\title{
Automorphism groups of linearly ordered structures and endomorphisms of the ordered set $(\mathbb{Q}, \leqslant)$ of rational numbers
}

\author{
Jillian D. McPhee, James D. Mitchell \& Martyn Quick \\ Mathematical Institute, University of St Andrews, North Haugh, \\ St Andrews, Fife, KY16 9SS, United Kingdom
}

July 13, 2016

\begin{abstract}
We investigate the structure of the monoid of endomorphisms of the ordered set $(\mathbb{Q}, \leqslant)$ of rational numbers. We show that for any countable linearly ordered set $\Omega$, there are uncountably many maximal subgroups of $\operatorname{End}(\mathbb{Q}, \leqslant)$ isomorphic to the automorphism group of $\Omega$. We characterise those subsets $X$ of $\mathbb{Q}$ that arise as a retract in $(\mathbb{Q}, \leqslant)$ in terms of topological information concerning $X$. Finally, we establish that a countable group arises as the automorphism group of a countable linearly ordered set, and hence as a maximal subgroup of $\operatorname{End}(\mathbb{Q}, \leqslant)$, if and only if it is free abelian of finite rank.
\end{abstract}

\section{Introduction}

The linearly ordered set $(\mathbb{Q}, \leqslant)$ of rational numbers has been observed to have a number of interesting properties. From the model theory point of view, $(\mathbb{Q}, \leqslant)$ is the Fraïssé limit of the class of finite linearly ordered sets. In addition, the automorphism group of $(\mathbb{Q}, \leqslant)$ is highly homogeneous as a permutation group on $\mathbb{Q}$ and it is oligomorphic (see, for example, [1, Section 9.3]). In this paper, we present an investigation into the structure of the endomorphism monoid of $(\mathbb{Q}, \leqslant)$. This continues our work begun in [3], where we examined the endomorphism monoid of the random graph and other related relational structures that arise as Fraïssé limits of various types of finite graph. The content of this paper then presents the beginnings of a counterpart within the study of the monoid of endomorphisms of $(\mathbb{Q}, \leqslant)$ to the literature on its automorphism group.

Our main result (Theorem A below) is to describe the groups that arise as maximal subgroups of the endomorphism monoid of $(\mathbb{Q}, \leqslant)$. The identity element in each such maximal subgroup $G$ is an idempotent endomorphism $f$ of $(\mathbb{Q}, \leqslant)$ and the group $G$ is then equal to the group $\mathscr{H}$-class of $f$. We identify the group $G$, up to isomorphism, from the structure of the image of $f$ (see Lemma 2.3 below). It is therefore important to identify which linearly ordered sets arise as retracts (that is, the image of an idempotent endomorphism) in $(\mathbb{Q}, \leqslant)$ and, within the first author's thesis [12], the original proof of Theorem A relied upon a precise description of such retracts as subsets of $\mathbb{Q}$. Recently, Kubiś [11] has studied retracts of Fraïssé limits in wider generality and he establishes the following theorem.

Theorem 1.1 (Kubiś, [11, Corollary 3.24]) Every countable linearly ordered set is order-isomorphic to an (increasing) retract of the set of rational numbers. 
("Increasing" in this statement merely refers to the fact that Kubiś uses the term "increasing map" for what we call an order-preserving map.) We shall use this result of Kubiś throughout Section 3 and it will enable us to present considerably shorter proofs than otherwise possible, essentially because we can always start with some endomorphism of $(\mathbb{Q}, \leqslant)$ with image order-isomorphic to some particular linearly ordered set.

Our main result is the following where we describe the groups arising as the maximal subgroups of $\operatorname{End}(\mathbb{Q}, \leqslant)$. There are some obvious restrictions on such groups: they must act as automorphisms on the image of the corresponding retract; i.e., they must be an automorphism group of some linearly ordered set. In the theorem, we observe that this is the only restriction. Moreover, we also show that each group occurs uncountably many times as a maximal subgroup of $\operatorname{End}(\mathbb{Q}, \leqslant)$. (Note that the trivial group occurs as the group $\mathscr{H}$-class of many idempotent endomorphisms including all those with finite image.)

Theorem A (i) Let $\Omega$ be a countable linearly ordered set. Then there exist $2^{\aleph_{0}}$ distinct regular $\mathscr{D}$-classes of $\operatorname{End}(\mathbb{Q}, \leqslant)$ whose group $\mathscr{H}$-classes are isomorphic to Aut $\Omega$.

(ii) There is one countable regular $\mathscr{D}$-class $D_{0}$ of $\operatorname{End}(\mathbb{Q}, \leqslant)$. This $D_{0}$ consists of the (idempotent) endomorphisms with image of cardinality 1 and every $\mathscr{H}$-class in $D_{0}$ is a group $\mathscr{H}$-class isomorphic to the trivial group.

All other regular $\mathscr{D}$-class of $\operatorname{End}(\mathbb{Q}, \leqslant)$ contain $2^{\aleph_{0}}$ distinct group $\mathscr{H}$-classes.

Green's relations are used to describe the structure of a semigroup. We describe them in more detail in Section 2 below. The $\mathscr{D}$-relation is the coarsest of those that we consider, while $\mathscr{D}$-classes are refined into $\mathscr{L}$ - and $\mathscr{R}$-classes. Finally $\mathscr{H}=\mathscr{L} \cap \mathscr{R}$. Maximal subgroups of a monoid are found as the group $\mathscr{H}$-classes within regular $\mathscr{D}$-classes and the idempotent elements within these classes play the role of the identity element in each maximal subgroup.

The strategy for proving Theorem A is quite similar to the corresponding theorems for Fraïssé limits of graphs in [3]. We shall construct uncountably many linearly ordered sets $\mathscr{C}_{\mathbf{x}}$ with trivial automorphism group that we "attach" to the linearly ordered set $\Omega$ in such a way that the result has automorphism group isomorphic to that of $\Omega$. There are two differences to note. The first is that the construction of the ordered set $\mathscr{C}_{\mathbf{x}}$ is relatively delicate, whereas the graph $L_{\Sigma}$ with trivial automorphism group in [3] is quite easy to build. On the other hand, having constructed $\mathscr{C}_{\mathbf{x}}$, the use of Kubiś's result makes it now straightforward to find an idempotent endomorphism with specified image.

We shall also establish further structural information concerning the endomorphism monoid of $(\mathbb{Q}, \leqslant)$ and its elements, as follows:

- We determine the number of $\mathscr{R}$ - and $\mathscr{L}$-classes in each $\mathscr{D}$-class and observe that this depends upon the cardinality of the image of any endomorphism within the $\mathscr{D}$-class (Theorems 3.7 and 3.9).

- If an endomorphism has finite image then it is a regular element of $\operatorname{End}(\mathbb{Q}, \leqslant)$ (Proposition 3.8), while non-regular endomorphisms can be constructed with certain types of infinite image (Theorem 3.10) from which it follows there are uncountably many non-regular $\mathscr{D}$-classes (Corollary 3.11).

Note that the first of these sets of observations applies to all $\mathscr{D}$-classes in $\operatorname{End}(\mathbb{Q}, \leqslant)$, which stands in contrast to our work in [3], where we established similar results for all regular $\mathscr{D}$-classes in the endomorphism monoid of the random graph and constructed some examples of non-regular $\mathscr{D}$-classes with uncountably many $\mathscr{R}$ - and $\mathscr{L}$-classes. 
Kubiś's result states that every countable linear ordered set is isomorphic to the image of some idempotent endomorphism of $(\mathbb{Q}, \leqslant)$. There is still the question of which subsets of $\mathbb{Q}$ actually arise as the image of an idempotent endomorphism. This is the content of the second theorem we state in this Introduction, where we characterise precisely which subsets are the images of idempotent endomorphisms. This theorem was used in the original proof of Theorem A as it appeared in [12]. Although no longer needed for the proofs in Section 3, this characterisation still seems to be of interest, particularly in the context of Theorem 1.1 quoted above from [11].

To state the theorem, we need the following concept. If $A$ is a subset of $\mathbb{Q}$, define a symmetric relation $\sim$ on $A$ as follows. If $a, b \in A$ with $a \leqslant b$, define $a \sim b$ if $c \in A$ for all $c$ satisfying $a \leqslant c \leqslant b$. It is straightforward to verify that $\sim$ is an equivalence relation on $A$. We shall call the equivalence classes of $A$ under this relation the maximal intervals of $A$. Indeed, it follows from the definition that every equivalence class of $\sim$ is an interval (as defined in Section 2 below) contained within $A$ and if $J$ is any interval contained in $A$ then $a \sim b$ for all $a, b \in J$ and hence $J$ is contained within one of the equivalence classes.

Theorem B Let $X$ be a subset of $\mathbb{Q}$. Then there is an idempotent endomorphism of $(\mathbb{Q}, \leqslant)$ with image equal to $X$ if and only if no maximal interval within $\mathbb{Q} \backslash X$ is closed (in the topology on $\mathbb{Q}$ induced from the Euclidean topology on $\mathbb{R}$ ).

Finally, we consider the question of which groups can arise as the automorphism group of a countable linearly ordered set; that is, which groups can arise as the maximal subgroup of $\operatorname{End}(\mathbb{Q}, \leqslant)$. In the case of graphs, it is known by Frucht's Theorem [5], together with the extension to infinite groups by de Groot [2] and Sabidussi [13], that every countable group arises as the automorphism group of a countable graph. In the case of linearly ordered structures, it is easily determined from the definition that there can be no nontrivial elements of finite order within the automorphism group of a linearly ordered set. On the other hand, such automorphism groups can, of course, be rather complicated. As an example, $\operatorname{Aut}(\mathbb{Q}, \leqslant)$ is an uncountable non-abelian group. Although we do not find a characterisation of all such groups (and it is unlikely that one exists), we do show that the structure of a countable group that arises as the automorphism group of a countable linearly ordered set is considerably constrained as the following theorem indicates.

Theorem $\mathbf{C}$ Let $(\Omega, \leqslant)$ be a countable totally ordered set and assume that $\operatorname{Aut}(\Omega, \leqslant)$ is countable. Then $\operatorname{Aut}(\Omega, \leqslant)$ is a free abelian group of finite rank.

The structure of this paper is as follows. In Section 2, we introduce all the basic notation and terminology that we require when discussing linearly ordered sets. We also recall the semigroup theory that we require, including stating results from our previous paper [3] that we use. Section 3 contains information about the structure of $\operatorname{End}(\mathbb{Q}, \leqslant)$. We prove Theorem A and establish the results concerning the $\mathscr{L}$ - and $\mathscr{R}$-classes in this monoid. Sections 4 and 5 are devoted to the proofs of Theorems B and C, respectively.

\section{Preliminaries and notation}

A relational structure is a pair $\Gamma=(V, \mathcal{E})$ consisting of a non-empty set $V$ and a sequence $\mathcal{E}=\left(E_{i}\right)_{i \in I}$ of relations on $V$. All the examples in this paper of relational structure will involve binary relations $E_{i}$ and will mostly be linearly ordered sets $(V, \leqslant)$, which is where $\leqslant$ is a reflexive, transitive and anti-symmetric relation on $V$ such that for every pair $u, v \in V$ either $u \leqslant v$ or $v \leqslant u$. In view of this, throughout we shall refer only to binary relations below although some definitions could be made in greater generality. 
If $\Gamma=\left(V,\left(E_{i}\right)_{i \in I}\right)$ and $\Delta=\left(W,\left(F_{i}\right)_{i \in I}\right)$ are relational structures (with relations indexed by the same set $I$ ), a homomorphism $f: \Gamma \rightarrow \Delta$ is a map $f: V \rightarrow W$ such that $(u f, v f) \in F_{i}$ whenever $(u, v) \in E_{i}$. In the case of linearly ordered sets, we shall also use the term order-preserving map as a synonym for homomorphism. Thus, if $\Gamma=(V, \leqslant)$ and $\Delta=(W, \leqslant)$ are linearly ordered sets, a map $f: V \rightarrow W$ defines a order-preserving map $\Gamma \rightarrow \Delta$ if $u \leqslant v$ in $\Gamma$ implies $u f \leqslant v f$ in $\Delta$. Note here that we are following the convention of writing maps on the right so that the image of a point $v \in V$ under a map is denoted by $v f$. The image of a order-preserving map $f:(V, \leqslant) \rightarrow(W, \leqslant)$ is the linearly ordered set $(V f, \leqslant)$ induced on the set of image values of $f$. The kernel of $f$ is the equivalence relation $\{(u, v) \in V \times V \mid u f=v f\}$. For each image value $x$ of $f$, the associated kernel class is the preimage $x f^{-1}=\{v \in V \mid v f=x\}$ and this is, of course, one of the equivalence classes defined by the kernel.

Furthermore, in our context an order-isomorphism is an isomorphism between two ordered sets. The term order-embedding is used to refer to a map $f:(V, \leqslant) \rightarrow(W, \leqslant)$ between two ordered sets such that $v \leqslant w$ in $(V, \leqslant)$ if and only if $v f \leqslant w f$ in $(W, \leqslant)$. It follows from this definition that $f$ is injective, but one should note that in the case of linearly ordered sets $(V, \leqslant)$ and $(W, \leqslant)$ an injective order-preserving map is always an order-embedding.

A linearly ordered set $(V, \leqslant)$ will be called dense if for every pair $u, v \in V$ with $u<v$ there exists some $w \in V$ satisfying $u<w<v$. If $A$ is a subset of $V$, a maximum element of $A$ is some $v \in A$ such that $a \leqslant v$ for all $a \in A$. The concept of minimum element is defined dually.

If $\Gamma=(V, \leqslant)$ is a linearly ordered set, a subset $U$ of $V$ is called convex if whenever $u, v$ and $w$ are points in $V$ with $u<w<v$ and $u, v \in U$ then necessarily $w \in U$ also. Note that the kernel class $x f^{-1}$ of a point in the image of a order-preserving map $f:(V, \leqslant) \rightarrow(W, \leqslant)$ is always convex. Other examples of convex subsets of $\Gamma$ include intervals. We shall borrow the standard notation used for intervals in the real line for intervals in $\Gamma$ :

$$
\begin{aligned}
{[u, v] } & =\{x \in V \mid u \leqslant x \leqslant v\} \\
(u, v) & =\{x \in V \mid u<x<v\}
\end{aligned}
$$

with $(u, v]$ and $[u, v)$ being defined similarly for points $u, v \in V$ with $u \leqslant v$. A further point to observe is that if $A$ and $B$ are disjoint convex subsets of $\Gamma$, then it must be the case that either $a<b$ for all $a \in A$ and $b \in B$, or that $a>b$ for all such $a$ and $b$. We shall write $A<B$ or $A>B$, respectively, to indicate these situations. Similarly, we shall use the notation $a<B$ as a short-hand to mean that the element $a$ satisfies $a<b$ for all $b \in B$.

The class of finite linearly ordered sets possesses the hereditary property, the joint embedding property and the amalgamation property. This class therefore has a unique Fraïssé limit [4] (see, for example, [8, Theorem 6.1.2]). It is well-known that this Fraïssé limit is the ordered set of rational numbers $(\mathbb{Q}, \leqslant)$. Indeed, this structure is the unique countable dense linearly ordered set with no maximum or minimum elements, in the sense that any linearly ordered set satisfying these properties is order-isomorphic to $(\mathbb{Q}, \leqslant)$.

At certain points, it will be helpful to view the set of ordered rational numbers $(\mathbb{Q}, \leqslant)$ as a substructure of the extended real numbers; that is, the relational structure $\mathbb{R}^{*}=$ $(\mathbb{R} \cup\{ \pm \infty\}, \leqslant)$ where $\leqslant$ denotes the usual order on $\mathbb{R}$ together with $-\infty \leqslant x \leqslant \infty$ for all $x \in \mathbb{R}$. We shall then extend the above definitions of intervals to include non-rational endpoints. Thus, for example, if $p, q \in \mathbb{R}^{*}$, we will be able to write $(p, q)$ for the interval in $\mathbb{Q}$ consisting of all rational numbers $x$ with $p<x<q$. Note by this notation we are always referring to a subset of $\mathbb{Q}$ but that the endpoints are permitted to be selected from 
outside of $\mathbb{Q}$. In an attempt to avoid confusion, we shall never use the interval notation to refer to subsets of $\mathbb{R}$.

Note that if $p$ or $q$ is not rational, then some of the intervals $(p, q),[p, q],(p, q]$ and $[p, q)$ coincide. In addition, our statement of Theorem B above refers to specific intervals in $\mathbb{Q}$ as being closed in the topology induced on $\mathbb{Q}$ from the usual topology on $\mathbb{R}$. Note that an interval in $\mathbb{Q}$ is closed in this induced topology precisely when it can be written in the form $[p, q]$ where $p, q \in \mathbb{R} \cup\{ \pm \infty\}$. In particular, the open interval $(p, q)$ is closed if and only if $p, q \in \mathbb{R} \backslash \mathbb{Q}$. When establishing the theorem in Section 4 below, we shall make use of this description of closed intervals in $\mathbb{Q}$.

One of the properties of the relational structure $\mathbb{R}^{*}$ is that a non-empty subset $A$ possesses a (possibly infinite) infimum and supremum. We shall write $\inf A$ and $\sup A$, as usual, to denote these elements and we shall use these mostly for subsets $A$ of $\mathbb{Q}$, but understand that in many cases $\inf A$ and $\sup A$ will be non-rational elements of $\mathbb{R}^{*}$. Using these constructions, though, one quickly observes that if $A$ is a convex subset of $\mathbb{Q}$, then $A$ equals one of the intervals $(p, q),[p, q),(p, q]$ or $[p, q]$ where $p=\inf A$ and $q=\sup A$ are some points of $\mathbb{R}^{*}$.

Let $M=$ End $\Gamma$ be the endomorphism monoid of a linearly ordered set $\Gamma=(V, \leqslant)$. We say that two elements $f$ and $g$ of $M$ are $\mathscr{L}$-related if $f$ and $g$ generate the same left ideal (that is, $M f=M g$ ). They are $\mathscr{R}$-related if $f M=g M$. Green's $\mathscr{H}$-relation is the intersection of the binary relations $\mathscr{L}$ and $\mathscr{R}$, and the $\mathscr{D}$-relation is the composite $\mathscr{L} \circ \mathscr{R}$ (which is also always an equivalence relation on $M$ ). The relevance of these relations to the study of subgroups contained within the endomorphism monoid is that if $e$ is an idempotent in $M$ (that is, if $e^{2}=e$ ) then the $\mathscr{H}$-class of $e$ is a subgroup of $M \quad[10$, Corollary 2.2.6] and, morever, the collection of maximal subgroups of $M$ are precisely the $\mathscr{H}$-classes of idempotents of $M$. We use the term group $\mathscr{H}$-class to refer to such maximal subgroups.

The following lemma is stated in greater generality for relational structures in our previous paper [3]. The first two parts are inherited from information concerning the $\mathscr{L}$ - and $\mathscr{R}$-classes of the full transformation monoid $\mathcal{T}_{V}$ of all maps $V \rightarrow V$ (see [10, Exercise 2.6.16]). Our restatement here is simply interpreting that lemma in the context of linearly ordered sets.

Lemma 2.1 ([3, Lemma 2.3]) Let $f$ and $g$ be endomorphisms of the linearly ordered set $\Gamma=(V, \leqslant)$.

(i) If $f$ and $g$ are $\mathscr{L}$-related, then $V f=V g$.

(ii) If $f$ and $g$ are $\mathscr{R}$-related, then $\operatorname{ker} f=\operatorname{ker} g$.

(iii) If $f$ and $g$ are $\mathscr{D}$-related, then $\operatorname{im} f$ and $\operatorname{im} g$ are order-isomorphic.

An element $f$ in the endomorphism monoid $M$ is said to be regular if there exists $g \in M$ such that $f g f=f$. An idempotent endomorphism $e$ is regular because $e^{3}=e$ and it is known that if $f$ is regular, then every element in the $\mathscr{D}$-class of $f$ is also regular $[10$, Proposition 2.3.1]. As noted in [3], in the case of regular endomorphisms the implications in Lemma 2.1 reverse.

Lemma 2.2 ([3, Lemma 2.5]) Let $f$ and $g$ be regular elements in the endomorphism monoid of the linearly ordered set $\Gamma=(V, \leqslant)$. Then

(i) $f$ and $g$ are $\mathscr{L}$-related if and only if $V f=V g$. 
(ii) $f$ and $g$ are $\mathscr{R}$-related if and only if $\operatorname{ker} f=\operatorname{ker} g$.

(iii) $f$ and $g$ are $\mathscr{D}$-related if and only if $\operatorname{im} f$ and $\operatorname{im} g$ are order-isomorphic.

The final fact that we require from our earlier work is that we can identify the group $\mathscr{H}$-classes in End $\Gamma$ as the automorphism group of the image of endomorphisms within the class.

Lemma 2.3 ([3, Proposition 2.6(iii)]) Let $f$ be an idempotent endomorphism of the linearly ordered set $\Gamma=(V, \leqslant)$. Then the group $\mathscr{H}$-class $H_{f}$ is isomorphic to the automorphism group of the image of $f$.

\section{The structure of $\operatorname{End}(\mathbb{Q}, \leqslant)$}

This section is devoted to the study of the endomorphism monoid of the ordered set $(\mathbb{Q}, \leqslant)$ of rational numbers. The first stage is to prove Theorem A concerning the group $\mathscr{H}$-classes within $\operatorname{End}(\mathbb{Q}, \leqslant)$. In preparation for the proof, we establish information concerning the number of idempotent endomorphisms with specified image (Theorem 3.1) and construct a family of linearly ordered sets with trivial automorphism group (Proposition 3.5). The remainder of the section is then concerned with establishing our information about $\mathscr{L}$ and $\mathscr{R}$-classes and about non-regular $\mathscr{D}$-classes in $\operatorname{End}(\mathbb{Q}, \leqslant)$.

Theorem 3.1 Let $\Omega=(X, \leqslant)$ be any countable linearly ordered set. Then

(i) if $|X|=1$, there are $\aleph_{0}$ idempotent endomorphisms $f$ of $(\mathbb{Q}, \leqslant)$ such that im $f \cong \Omega$;

(ii) if $|X|>1$, there are $2^{\aleph_{0}}$ idempotent endomorphisms $f$ of $(\mathbb{Q}, \leqslant)$ such that $\operatorname{im} f \cong \Omega$.

Proof: (i) An endomorphism with image of cardinality 1 has the form $x f=q$ for all $x \in \mathbb{Q}$, where $q$ is some fixed point in $\mathbb{Q}$. All such endomorphisms are idempotent and there are countably many such maps.

(ii) Suppose $|X|>1$. By Theorem 1.1, there exists an idempotent endomorphism $f$ of $(\mathbb{Q}, \leqslant)$ with $\operatorname{im} f \cong \Omega$. Choose some $q \in \operatorname{im} f$ subject to the condition that $q$ is not the maximum element of the image and let $I=q f^{-1}$. Put $\alpha=\inf I$ and $\beta=\sup I$, which are defined elements of the extended real numbers $\mathbb{R}^{*}$. By our assumption, $\beta<$ $\infty$. We shall define an idempotent endomorphism $g_{\gamma}$ of $(\mathbb{Q}, \leqslant)$ as $g_{\gamma}=\xi f \eta$ in terms of certain $\xi, \eta \in \operatorname{End}(\mathbb{Q}, \leqslant)$. The definition of the latter will depend upon the choice of the parameter $\gamma \geqslant \beta$, but will also be different according to whether or not $\beta \in \mathbb{Q}$ and whether $\beta$ lies in the interval $I$. The maps $\xi$ and $\eta$ will be arranged so that $\eta \xi$ is the identity map and the image of $g_{\gamma}$ is also isomorphic to $\Omega$.

Case 1: $\boldsymbol{\beta} \notin \mathbb{Q}$. In this case, we first choose any $\gamma \in \mathbb{R} \backslash \mathbb{Q}$ with with $\gamma \geqslant \beta$. Note that there are uncountably many possible choices for such $\gamma$. Since $\beta \notin \mathbb{Q}$, necessarily $\alpha<\beta$, so we may also choose some $\delta \in \mathbb{R} \backslash \mathbb{Q}$ satisfying $\alpha<\delta<\beta$. The intervals $(\delta, \beta),(\delta, \gamma)$, $(\beta, \infty)$ and $(\gamma, \infty)$ are all order-isomorphic to $(\mathbb{Q}, \leqslant)$ and so there are order-isomorphisms $\theta_{1}:(\delta, \beta) \rightarrow(\delta, \gamma)$ and $\theta_{2}:(\beta, \infty) \rightarrow(\gamma, \infty)$. Define $\xi$ to be the order-automorphism of $(\mathbb{Q}, \leqslant)$ given by

$$
x \xi= \begin{cases}x & \text { for } x<\delta \\ x \theta_{1} & \text { for } \delta<x<\beta \\ x \theta_{2} & \text { for } x>\beta\end{cases}
$$

and $\eta$ to be its inverse. Certainly then $\eta \xi$ is the identity map on $\mathbb{Q}$ and $I \xi^{-1}=I \cup(\beta, \gamma)$ (that is, $I \xi^{-1}=(\alpha, \gamma)$ or $[\alpha, \gamma)$, depending upon whether or not $\left.\alpha \in I\right)$. 
Case 2: $\boldsymbol{\beta} \in \mathbb{Q}$ and $\boldsymbol{\beta} \in \boldsymbol{I}$. In this case, consider any $\gamma \in \mathbb{R}$ with $\gamma \geqslant \beta$. We use an order-isomorphism $\theta$ from $(\gamma, \infty)$ to $(\beta, \infty)$, to define $\xi, \eta \in \operatorname{End}(\mathbb{Q}, \leqslant)$ by

$$
x \xi= \begin{cases}x & \text { for } x \leqslant \beta \\ \beta & \text { for } \beta<x \leqslant \gamma \\ x \theta & \text { for } x>\gamma\end{cases}
$$

and

$$
x \eta= \begin{cases}x & \text { for } x \leqslant \beta \\ x \theta^{-1} & \text { for } x>\beta .\end{cases}
$$

Then by construction, $\eta \xi$ is the identity map on $\mathbb{Q}$ and $I \xi^{-1}=I \cup(\beta, \gamma]$ (that is, $I \xi^{-1}=$ $(\alpha, \gamma]$ or $[\alpha, \gamma]$, depending upon whether or not $\alpha \in I)$.

Case 3: $\boldsymbol{\beta} \in \mathbb{Q}$ but $\boldsymbol{\beta} \notin \boldsymbol{I}$. Again we consider any $\gamma \in \mathbb{R}$ with $\gamma \geqslant \beta$. We pick some $\delta \in \mathbb{Q}$ with $\delta>\gamma$ and let $\theta_{1}:(\alpha, \gamma) \rightarrow(\alpha, \beta)$ and $\theta_{2}:(\delta, \infty) \rightarrow(\beta, \infty)$ be orderisomorphisms. Now define $\xi, \eta \in \operatorname{End}(\mathbb{Q}, \leqslant)$ by

$$
x \xi= \begin{cases}x & \text { for } x \leqslant \alpha \\ x \theta_{1} & \text { for } \alpha<x<\gamma \\ \beta & \text { for } \gamma \leqslant x \leqslant \delta \\ x \theta_{2} & \text { for } x>\delta\end{cases}
$$

and

$$
x \eta= \begin{cases}x & \text { for } x \leqslant \alpha \\ x \theta_{1}^{-1} & \text { for } \alpha<x<\beta \\ \delta & \text { for } x=\beta \\ x \theta_{2}^{-1} & \text { for } x>\beta .\end{cases}
$$

Then $\eta \xi$ is the identity map on $\mathbb{Q}$ and $I \xi^{-1}=I \cup[\beta, \gamma)$ (that is, $I \xi^{-1}=(\alpha, \gamma)$ or $[\alpha, \gamma)$, depending upon whether or not $\alpha \in I$ ).

Using the $\xi$ and $\eta$ just defined (depending upon, amongst other things, a choice of $\gamma$ ), write $g_{\gamma}=\xi f \eta$. Using the fact that $f^{2}=f$ and $\eta \xi$ is the identity, we observe that $g_{\gamma}$ is also an idempotent endomorphism of $(\mathbb{Q}, \leqslant)$. As $\xi$ is surjective and $\eta$ is injective in each case, it follows that $\operatorname{im} g_{\gamma}=\operatorname{im} f \eta \cong \operatorname{im} f \cong \Omega$. Moreover, $q g_{\gamma}^{-1}=I \xi^{-1}$ and this equals some interval $J$ with $(\alpha, \gamma) \subseteq J \subseteq[\alpha, \gamma]$.

In conclusion, as in each case there are uncountably many choices for $\gamma$, we have constructed $2^{\aleph_{0}}$ idempotent endomorphisms $g_{\gamma}$ with image isomorphic to $\Omega$.

We shall now embark upon the proof of our main theorem. The first step is to construct some linearly ordered sets with trivial automorphism group.

Consider an enumeration $\mathbf{x}=\left(x_{n}\right)$ of the set $\mathbb{Q}$ of rational numbers. Define a set $C_{\mathbf{x}}$ depending upon this enumeration as a set of ordered pairs of rational numbers and integers as follows:

$$
C_{\mathbf{x}}=\left\{\left(x_{n}, i\right) \mid n \in \mathbb{N}, 0 \leqslant i \leqslant n\right\} .
$$

We write $\mathscr{C}_{\mathbf{x}}=\left(C_{\mathbf{x}}, \leqslant\right)$ for the linearly ordered set where $\leqslant$ is the lexicographic order on $C_{\mathbf{x}}$ :

$$
\left(x_{m}, i\right) \leqslant\left(x_{n}, j\right) \quad \text { if and only if } \quad \text { either } x_{m}<x_{n} \text {, or both } m=n \text { and } i \leqslant j .
$$


Essentially this definition arranges the points in the set $X_{n}=\left\{\left(x_{n}, i\right) \mid 0 \leqslant i \leqslant n\right\}$ in increasing order as indexed by $i$ and then orders the sets $X_{n}$ relative to each other according to the linear order on $\mathbb{Q}$. Thus, we are in effect constructing $\mathscr{C}_{\mathbf{x}}$ from $(\mathbb{Q}, \leqslant)$ by replacing each point $x_{n}$ in $\mathbb{Q}$ by a finite chain of length $n$.

It is a straightforward observation, using the fact that $(\mathbb{Q}, \leqslant)$ is linearly ordered, to observe that $\mathscr{C}_{\mathbf{x}}$ is also a linearly ordered set. Furthermore, we similarly deduce the following facts.

Lemma 3.2 Consider two points $\left(x_{m}, i\right),\left(x_{n}, j\right) \in C_{\mathbf{x}}$.

(i) There are infinitely many $c \in C_{\mathbf{x}}$ and infinitely many $d \in C_{\mathbf{x}}$ such that $c<\left(x_{m}, i\right)<$ $d$.

(ii) If $x_{m}<x_{n}$, then there exist infinitely many $c \in C_{\mathbf{x}}$ such that $\left(x_{m}, i\right)<c<\left(x_{n}, j\right)$.

(iii) For every $i$ with $0 \leqslant i<n-1$, there exist no element $c \in C_{\mathbf{x}}$ such that $\left(x_{n}, i\right)<c<$ $\left(x_{n}, i+1\right)$.

Lemma 3.3 Let $\mathbf{x}=\left(x_{n}\right)$ and $\mathbf{y}=\left(y_{n}\right)$ be two enumerations of $\mathbb{Q}$. Then $\mathscr{C}_{\mathbf{x}}=\left(C_{\mathbf{x}}, \leqslant\right)$ and $\mathscr{C}_{\mathbf{y}}=\left(C_{\mathbf{y}}, \leqslant\right)$ are order-isomorphic if and only if the map $x_{n} \mapsto y_{n}$, for $n \in \mathbb{N}$, defines an automorphism of the ordered set $(\mathbb{Q}, \leqslant)$. Specifically, if $\phi$ is an order-isomorphism from $\mathscr{C}_{\mathbf{x}}$ to $\mathscr{C}_{\mathbf{y}}$, then $\left(x_{n}, i\right) \phi=\left(y_{n}, i\right)$ for all $n \in N$ and $0 \leqslant i \leqslant n$.

Proof: Suppose $\phi$ is an order-isomorphism from $\mathscr{C}_{\mathbf{x}}$ to $\mathscr{C}_{\mathbf{y}}$. Then for $n \in \mathbb{N}$, write $X_{n}=\left\{\left(x_{n}, i\right) \mid 0 \leqslant i \leqslant n\right\}$ and $Y_{n}=\left\{\left(y_{n}, i\right) \mid 0 \leqslant i \leqslant n\right\}$. We shall first observe that, for each $n \in \mathbb{N}$, there exists some $m$ with $X_{n} \phi \subseteq Y_{m}$. Suppose, for a contradiction, that $X_{n} \phi \nsubseteq Y_{m}$ for all $m \in \mathbb{N}$. The elements $\left(x_{n}, i\right)$, for $0 \leqslant i \leqslant n$, are then mapped into at least two different sets $Y_{m}$ and so there is some value $k$ such that $\left(x_{n}, k\right) \phi \in Y_{m}$ and $\left(x_{n}, k+1\right) \phi \in Y_{m^{\prime}}$ for distinct values $m, m^{\prime} \in \mathbb{N}$. Since $\left(x_{n}, k\right) \phi<\left(x_{n}, k+1\right) \phi$, it must be the case that $y_{m}<y_{m^{\prime}}$. Now by Lemma 3.2(ii), there exists $c \in C_{\mathbf{y}}$ satisfying $\left(x_{n}, k\right) \phi<c<\left(x_{n}, k+1\right) \phi$. We then conclude $\left(x_{n}, k\right)<c \phi^{-1}<\left(x_{n}, k+1\right)$, which contradicts Lemma 3.2(iii).

In conclusion, there exists some $m$ such that $X_{n} \phi \subseteq Y_{m}$. However, $\phi^{-1}$ also defines an order-isomorphism from $\mathscr{C}_{\mathbf{y}}$ to $\mathscr{C}_{\mathbf{x}}$ and the set $Y_{m} \phi^{-1}$ contains all the points from $X_{n}$. The argument above applied to $\phi^{-1}$ then establishes that $Y_{m} \phi^{-1}=X_{n}$ and so $X_{n} \phi=Y_{m}$. Since $X_{n}$ contains precisely $n$ points, we conclude that $m=n$. Since $\phi$ is order-preserving, it now follows that $\left(x_{n}, i\right) \phi=\left(y_{n}, i\right)$ for all $n \in \mathbb{N}$ and $0 \leqslant i \leqslant n$, as claimed in the statement of the lemma. Now, if $x_{m} \leqslant x_{n}$, then it follows that $\left(x_{m}, 0\right) \phi \leqslant\left(x_{n}, 0\right) \phi$, so necessarily $y_{m} \leqslant y_{n}$. Hence we conclude that the map $x_{n} \mapsto y_{n}$ is indeed an automorphism of $(\mathbb{Q}, \leqslant)$.

Conversely, if $x_{n} \mapsto y_{n}$ is an automorphism of $(\mathbb{Q}, \leqslant)$, then the map $\left(x_{n}, i\right) \mapsto\left(y_{n}, i\right)$, for $1 \leqslant i \leqslant n$ and $n \in \mathbb{N}$, defines an order-isomorphism $\mathscr{C}_{\mathbf{x}} \rightarrow \mathscr{C}_{\mathbf{y}}$. This completes the proof of the lemma.

Taking $\mathbf{y}=\mathbf{x}$ in the formula for order-isomorphisms in the previous lemma yields:

Corollary 3.4 Let $\mathbf{x}=\left(x_{n}\right)$ be an enumeration of $\mathbb{Q}$ and $\mathscr{C}_{\mathbf{x}}=\left(C_{\mathbf{x}}, \leqslant\right)$. Then Aut $\mathscr{C}_{\mathbf{x}}$ is trivial.

Proposition 3.5 There exists a set $P$ of $2^{\aleph_{0}}$ many enumerations of the set $\mathbb{Q}$ of rational numbers such that $\mathscr{C}_{\mathbf{x}} \neq \mathscr{C}_{\mathbf{y}}$ for distinct $\mathbf{x}, \mathbf{y} \in P$. 
Proof: Fix one enumeration $\mathbf{x}=\left(x_{n}\right)$ of the set $\mathbb{Q}$. For each $i \in \mathbb{N}$, define $\pi_{i}$ to be the transposition $(2 i 2 i+1)$ in the symmetric group $\operatorname{Sym}(\mathbb{N})$ and, for any subset $A \subseteq \mathbb{N}$, define the involution $\pi_{A}=\prod_{i \in A} \pi_{i}$. We shall write $\mathbf{x} \pi_{A}$ for the enumeration $\left(x_{n \pi_{A}}\right)$ of $\mathbb{Q}$ and set $P=\left\{\mathbf{x} \pi_{A} \mid A \subseteq \mathbb{N}\right\}$. Note that, for distinct subsets $A, B \subseteq \mathbb{N}$, the map given by $x_{n \pi_{A}} \mapsto x_{n \pi_{B}}$ for $n \in \mathbb{N}$ cannot be an order-automorphism of $(\mathbb{Q}, \leqslant)$, since $\pi_{A} \pi_{B}$ is again an involution. It then follows that the ordered sets $\mathscr{C}_{\mathbf{x} \pi_{A}}$, for $A \subseteq \mathbb{N}$, are pairwise non-isomorphic by Lemma 3.3.

If $\Omega=\left(U, \leqslant_{1}\right)$ and $\Lambda=\left(V, \leqslant_{2}\right)$ are two linearly ordered sets, we can define a new ordered set, that we shall denote by $\Omega+\Lambda$, as $\Omega+\Lambda=(U \cup V, \leqslant)$, where we assume that the sets $U$ and $V$ are disjoint and where we define the order $\leqslant$ on $U \cup V$ by $v \leqslant w$ if and only if one of the following conditions holds (i) $v, w \in U$ and $v \leqslant 1$, (ii) $v, w \in V$ and $v \leqslant_{2} w$, or (iii) $v \in U$ and $w \in V$. In effect, in $\Omega+\Lambda$, we are retaining the order in both $\Omega$ and $\Lambda$ but in addition are placing all points in $\Omega$ before all points in $\Lambda$. One observes immediately that $\Omega+\Lambda$ is then also a linearly ordered set and is the union of two substructures isomorphic to $\Omega$ and to $\Lambda$, respectively.

Proposition 3.6 Let $\Omega=(V, \leqslant)$ be any linearly ordered set.

(i) If $\mathbf{x}$ is any enumeration of the set $\mathbb{Q}$ of rational numbers, then $\operatorname{Aut}\left(\Omega+\mathscr{C}_{\mathbf{x}}\right)$ is isomorphic to Aut $\Omega$.

(ii) If $\mathbf{x}$ and $\mathbf{y}$ are enumerations of $\mathbb{Q}$, then $\Omega+\mathscr{C}_{\mathbf{x}}$ is order-isomorphic to $\Omega+\mathscr{C}_{\mathbf{y}}$ if and only if $\mathscr{C}_{\mathbf{x}}$ is order-isomorphic to $\mathscr{C}_{\mathbf{y}}$.

Proof: (i) Recall that the set of points in $\Omega+\mathscr{C}_{\mathbf{x}}$ is the union $V \cup C_{\mathbf{x}}$. To simplify notation, we shall write $\leqslant$ for the order both on $\Omega$ and on $\Omega+\mathscr{C}_{\mathbf{x}}$, since they coincide for points in the set $V$. We shall first, using a variation of the argument employed in Lemma 3.3, show that if $f \in \operatorname{Aut}\left(\Omega+\mathscr{C}_{\mathbf{x}}\right)$ then $V f=V$ and $C_{\mathbf{x}} f=C_{\mathbf{x}}$. As before, we write $X_{n}$ for the subset $\left\{\left(x_{n}, i\right) \mid 0 \leqslant i \leqslant n\right\}$ of $C_{\mathbf{x}}$.

Case 1: We first consider the case when $C_{\mathbf{x}} f \subseteq C_{\mathbf{x}}$. Then for each $n \in N$, the set $X_{n}$ is mapped into $C_{\mathbf{x}}$ and the same argument as used in Lemma 3.3 shows that there exists some $m=m(n)$ such that $X_{n} f \subseteq X_{m}$. Note $m \geqslant n$ due to the cardinality of the two sets involved.

If $m>n$, there exists some $c \in X_{m} \backslash X_{n} f$. Pick some $d \in X_{n}$. Now either $d f<c$ or $c<d f$. We consider the case when $c<d f$. Now $c=v f$ for some $v \in V \cup C_{\mathbf{x}}$. We use parts (i) or (ii) of Lemma 3.2, depending upon whether $v \in V$ or $v \in C_{\mathbf{x}}$, to produce infinitely many elements $b \in C_{\mathbf{x}}$ satisfying $v<b<d$. Then $v f<b f<d f$, so $b f \in X_{n}$ also. This is a contradiction since $X_{n}$ is finite. When $d f<c$, the argument is identical (though we know immediately from the order on $\Omega+\mathscr{C}_{\mathbf{x}}$ that necessarily $c$ is the image of a point from $C_{\mathbf{x}}$ ). We conclude that $m(n)=n$ for all $n \in \mathbb{N}$ and so our map satisfies $X_{n} f=X_{n}$ for all $n \in \mathbb{N}$ and hence $C_{\mathbf{x}} f=C_{\mathbf{x}}$. It then follows that $V f=V$ also.

Case 2: Suppose that $C_{\mathbf{x}} f \nsubseteq C_{\mathbf{x}}$. Then there exists some $c \in C_{\mathbf{x}}$ such that $c f \in V$. Then if $d$ is any point in $C_{\mathbf{x}}$, it satisfies $c f<d$, so that $c<d f^{-1}$. Necessarily then $d f^{-1} \in C_{\mathbf{x}}$ and we deduce $d \in C_{\mathbf{x}} f$. We conclude that in this case $C_{\mathbf{x}} f \supseteq C_{\mathbf{x}}$. We then apply the inverse of $f$ and note that $f^{-1}$ is an automorphism of $\Omega+\mathscr{C}_{\mathrm{x}}$ that satisfies $C_{\mathbf{x}} f^{-1} \subseteq C_{\mathbf{x}}$. Case 1 tells us that $C_{\mathbf{x}} f^{-1}=C_{\mathbf{x}}$ and $V f^{-1}=V$. Hence $C_{\mathbf{x}} f=C_{\mathbf{x}}$ and $V f=V$, as claimed. 
Now that we know $C_{\mathbf{x}} f=C_{\mathbf{x}}$ and $V f=V$ for every automorphism $f$ of $\Omega+\mathscr{C}_{x}$ it is a simple matter to conclude that

$$
\operatorname{Aut}\left(\Omega+\mathscr{C}_{\mathbf{x}}\right) \cong \operatorname{Aut} \Omega \times \operatorname{Aut} \mathscr{C}_{x} \cong \operatorname{Aut} \Omega,
$$

with use of Corollary 3.4.

(ii) This is established similarly. We observe that if $\phi$ is an isomorphism from $\Omega+\mathscr{C}_{\mathbf{x}}$ to $\Omega+\mathscr{C}_{\mathbf{y}}$, then we show, using the argument just used in part (i), that $V \phi=V$ and $C_{\mathbf{x}} \phi=C_{\mathbf{y}}$. It then follows that $\phi$ induces an isomorphism $\mathscr{C}_{\mathbf{x}} \rightarrow \mathscr{C}_{\mathbf{y}}$.

We are now able to prove our main theorem concerning the maximal subgroups of $\operatorname{End}(\mathbb{Q}, \leqslant)$ as stated in the Introduction.

Proof of Theorem A: (i) Let $\Omega$ be any countable linearly ordered set. Let $P$ be a set of $2^{\aleph_{0}}$ many enumerations of $\mathbb{Q}$ such that $\mathscr{C}_{\mathbf{x}} \neq \mathscr{C}_{\mathbf{y}}$ when $\mathbf{x}$ and $\mathbf{y}$ are distinct members of $P$, as provided by Proposition 3.5. Now if $\mathrm{x} \in P$, then $\Omega+\mathscr{C}_{\mathbf{x}}$ is some countable linearly ordered set and so, by Theorem $1.1, \Omega+\mathscr{C}_{\mathbf{x}}$ is isomorphic to some retract of $(\mathbb{Q}, \leqslant)$; that is, there is an idempotent endomorphism $f_{\mathbf{x}}$ of $(\mathbb{Q}, \leqslant)$ such that im $f_{\mathbf{x}} \cong \Omega+\mathscr{C}_{\mathbf{x}}$. Then the $\mathscr{H}$-class of $f_{\mathrm{x}}$ is

$$
H_{f_{\mathbf{x}}} \cong \operatorname{Aut}\left(\Omega+\mathscr{C}_{\mathbf{x}}\right) \cong \operatorname{Aut} \Omega,
$$

by Proposition 3.6(i). Hence each $f_{\mathbf{x}}$ is an idempotent endomorphism with $\mathscr{H}$-class isomorphic to the automorphism group of $\Omega$.

Observe, moreover, that since $\Omega+\mathscr{C}_{\mathbf{x}} \neq \Omega+\mathscr{C}_{\mathbf{y}}$ for distinct $\mathbf{x}, \mathbf{y} \in P$ as shown in Proposition 3.6(ii), the $\mathscr{D}$-classes of the idempotent endomorphisms $f_{\mathbf{x}}$ are distinct, using Lemma 2.2(iii). Hence there are $2^{\aleph_{0}}$ distinct regular $\mathscr{D}$-classes of $\operatorname{End}(\mathbb{Q}, \leqslant)$ with group $\mathscr{H}$-class isomorphic to Aut $\Omega$.

(ii) We make use of Theorem 3.1. Part (i) of that theorem tells us that any endomorphism of $(\mathbb{Q}, \leqslant)$ with image of cardinality 1 is idempotent, therefore regular, and the set of all such endomorphisms forms a single $\mathscr{D}$-class $D_{0}$ by Lemma 2.2(iii). If $f \in D_{0}$, then $\{f\}$ is a single $\mathscr{H}$-class, again by use of Lemma 2.2 , since any two endomorphisms in $D_{0}$ are $\mathscr{R}$-related but no distinct pair are $\mathscr{L}$-related. Thus $H_{f}=\{f\}$ and this is a copy of the trivial group.

If $D$ is any other $\mathscr{D}$-class of $\operatorname{End}(\mathbb{Q}, \leqslant)$. Fix $f_{0} \in D$ and write $\Omega=\operatorname{im} f_{0}$. By Theorem 3.1(ii), there are $2^{\aleph_{0}}$ idempotent endomorphisms $f$ of $(\mathbb{Q}, \leqslant)$ with $\operatorname{im} f \cong \Omega$. Each such $f$ belongs to $D$ by Lemma 2.2(iii) and determine a distinct group $\mathscr{H}$-class $H_{f} \cong$ Aut $\Omega$ by Lemma 2.3. This completes the proof of the theorem.

The first paragraph of the proof of Theorem A(ii) above also establishes part (i) of our result about the $\mathscr{R}$-classes of $\operatorname{End}(\mathbb{Q}, \leqslant)$ as follows.

Theorem 3.7 Let $f$ be an endomorphism of $(\mathbb{Q}, \leqslant)$ and write $X=\operatorname{im} f$. Then

(i) if $|X|=1$, the $\mathscr{D}$-class of $f$ is a single $\mathscr{R}$-class;

(ii) if $|X|>1$, the $\mathscr{D}$-class of $f$ contains $2^{\aleph_{0}}$ many $\mathscr{R}$-classes.

Proof: (ii) Assume that $|X|>1$. Our argument is similar to that which establishes part (ii) of Theorem 3.1 above. Indeed, choose $q \in X$ that is not the maximum element of $X$, put $I=q f^{-1}, \quad \alpha=\inf I$ and $\beta=\sup I$. Choose $\gamma$ to be a suitable real number with $\gamma \geqslant \beta$ and then define maps $\xi$ and $\eta$ by the same formulae (depending upon whether 
$\beta \in \mathbb{Q}$ and whether $\beta \in I$ ) as found in the proof of Theorem 3.1. Then, as noted before, $\eta \xi$ is the identity map on $\mathbb{Q}$ and $(\alpha, \gamma) \subseteq I \xi^{-1} \subseteq[\alpha, \gamma]$.

Now the map $\xi f$ is $\mathscr{L}$-related to $f$ in view of the formula $\eta \xi f=f$. Note that the kernel class of all points in $\mathbb{Q}$ that map to $q$ under $\xi f$ equals $q(\xi f)^{-1}=I \xi^{-1}$, whose form is as described above. Thus as $\gamma$ varies, we obtain $2^{\aleph_{0}}$ endomorphisms in the $\mathscr{D}$-class of $f$ that are not $\mathscr{R}$-related to each other because they have distinct kernels.

Proposition 3.8 Let $f$ be any endomorphism of $(\mathbb{Q}, \leqslant)$. If $\operatorname{im} f$ is finite, then $f$ is regular.

Proof: Let $X=\operatorname{im} f$ and $x_{1}, x_{2}, \ldots, x_{n}$ be the distinct image values of $f$. Choose $q_{i} \in x_{i} f^{-1}$ for each $i$. There is an automorphism $g$ of $(\mathbb{Q}, \leqslant)$ satisfying $x_{i} g=q_{i}$ for each $i$. Then $g \in \operatorname{End}(\mathbb{Q}, \leqslant)$ and $f g f=f$. Hence $f$ is regular.

Theorem 3.9 Let $f$ be an endomorphism of $(\mathbb{Q}, \leqslant)$ and write $X=\operatorname{im} f$. Then

(i) if $X$ is finite, the $\mathscr{D}$-class of $f$ contains $\aleph_{0}$ many $\mathscr{L}$-classes;

(ii) if $X$ is infinite, the $\mathscr{D}$-class of $f$ contains $2^{\aleph_{0}}$ many $\mathscr{L}$-classes.

Proof: (i) First note that if $X$ is finite, then $f$ is regular by Proposition 3.8 and, indeed, by Lemma 2.2(iii), another endomorphism $g$ is $\mathscr{D}$-related to $f$ if and only if $|\operatorname{im} g|=|X|$. Given two endomorphisms $g$ and $h$ with images of the same cardinality, they are $\mathscr{L}$-related if and only if their images are equal (in addition to being isomorphic). There are countably many choices for a subset of $\mathbb{Q}$ of a particular finite cardinality and hence the $\mathscr{D}$-class of $f$ contains countably many $\mathscr{L}$-classes.

(ii) Now suppose that $X$ is infinite. We divide into two cases:

(a) Either $X$ contains an infinite sequence $\left(x_{n}\right)$ of points such that, for each $n, x_{n}$ is the maximum member of $X \backslash\left\{x_{1}, x_{2}, \ldots, x_{n-1}\right\}$,

(b) or there are finitely many points $x_{1}, x_{2}, \ldots, x_{n}$ in $X$ such that $x_{i}$ is the maximum member of $X \backslash\left\{x_{1}, x_{2}, \ldots, x_{i-1}\right\}$ and such that $X \backslash\left\{x_{1}, x_{2}, \ldots, x_{n}\right\}$ has no maximum member.

(When $X$ has no maximum element, we are in Case (b) with $n=0$.)

Suppose then that we are in Case (a). Put $Y=X \backslash\left\{x_{1}, x_{2}, \ldots\right\}$ and let $\alpha=\sup Y$. If $Y$ is empty, take $\alpha=-\infty$. Note then that $\alpha<x_{n}<x_{n-1}$ for all $n$. Now pick any rational number $q_{1}>\alpha$ and, having chosen $q_{1}, q_{2}, \ldots, q_{n-1}$, pick any rational number $q_{n}$ satisfying $\alpha<q_{n}<q_{n-1}$. There are $2^{\aleph_{0}}$ many ways of choosing the resulting sequence $\mathbf{q}=\left(q_{n}\right)$. Now $(\alpha, \infty)$ is order isomorphic to $\mathbb{Q}$ and hence there is an order-preserving bijection $\xi=\xi_{\mathbf{q}}$ from $(\alpha, \infty)$ to itself that maps $x_{n}$ to $q_{n}$ for each $n \in \mathbb{N}$. Extend this to an automorphism $\xi$ of $(\mathbb{Q}, \leqslant)$ by defining $x \xi=x$ for all $x \leqslant \alpha$. Then $f \xi$ is $\mathscr{R}$-related to $f$ and $\operatorname{im} f \xi=X \xi=Y \cup\left\{q_{1}, q_{2}, \ldots\right\}$. Consequently, by Lemma 2.1(i), all such $f \xi$ lie in different $\mathscr{L}$-classes and we have established the claimed result in this case.

We now turn to Case (b). Put $Y=X \backslash\left\{x_{1}, x_{2}, \ldots, x_{n}\right\}$ and let $\alpha=\sup Y$. (In this case, necessarily $Y$ is non-empty.) Note, by assumption, $\alpha \notin X$. Choose any real number $\beta$ with $\beta<\alpha$. Since the intervals $(-\infty, \alpha)$ and $(-\infty, \beta)$ are order-isomorphic, there is an order-isomorphism $\theta:(-\infty, \alpha) \rightarrow(-\infty, \beta)$. Pick any rational number $\gamma$ with $\alpha \leqslant \gamma \leqslant x_{n}$. Then define $\xi, \eta \in \operatorname{End}(\mathbb{Q}, \leqslant)$ by

$$
x \xi= \begin{cases}x \theta & \text { if } x<\alpha \\ x & \text { if } x \geqslant \alpha\end{cases}
$$


and

$$
x \eta= \begin{cases}x \theta^{-1} & \text { if } x<\beta \\ \gamma & \text { if } \beta \leqslant x \leqslant \gamma \\ x & \text { if } x \geqslant \gamma .\end{cases}
$$

Then $x_{i} \xi \eta=x_{i}$ for $i=1,2, \ldots, n$, since each $x_{i} \geqslant \gamma$, while $\xi \eta$ is the identity map on $Y$. We therefore conclude $f \xi \eta=f$. It follows that $f \xi$ and $f$ are $\mathscr{R}$-related. Moreover, $\operatorname{im} f \xi=X \xi=Y \theta \cup\left\{x_{1}, x_{2}, \ldots, x_{n}\right\}$ and $\sup Y \theta=\beta$. Hence, as $\beta$ is permitted to vary through $\{\beta \in \mathbb{R} \mid \beta<\alpha\}$, we obtain $2^{\aleph_{0}}$ endomorphisms in the $\mathscr{D}$-class of $f$, all of which belong to distinct $\mathscr{L}$-classes by Lemma 2.1(i). This completes the proof.

The following result provides a condition that is sufficient for producing non-regular endomorphisms. It is phrased in terms of the infimum and supremum of a subset of $\mathbb{Q}$. We remind the reader that these are well-defined members of $\mathbb{R}^{*}$ and might not necessarily be rational numbers in general.

Theorem 3.10 Let $X$ be a subset of $\mathbb{Q}$ with the property that $X$ has a partition into two disjoint subsets $X=X_{-} \cup X_{+}$where $X_{-}<X_{+}$and such that $\alpha=\sup X_{-}$and $\beta=\inf X_{+}$ do not belong to $X$. Then there exists a non-regular endomorphism $f$ of $(\mathbb{Q}, \leqslant)$ such that the image of $f$ is order-isomorphic to the substructure $(X, \leqslant)$.

Proof: We make a number of reductions. The first is to observe that we can assume that $X$ is the image of an endomorphism of $(\mathbb{Q}, \leqslant)$. Indeed, by Theorem 1.1, there is an (idempotent) endomorphism $g$ of $(\mathbb{Q}, \leqslant)$ with image isomorphic to $X$. Write $Y=\operatorname{im} g$ and denote by $\phi$ the order-isomorphism from $(X, \leqslant)$ to $(Y, \leqslant)$. Let $Y_{-}=X_{-} \phi$ and $Y_{+}=X_{+} \phi$. Put $\gamma=\sup Y_{-}$. If it were the case that $\gamma \in Y$, then $\gamma=x \phi$ for some $x \in X$. This element $x$ cannot be a member of $X_{-}$, since $x$ would then be the maximum element of $X_{-}$, contradicting the assuming that $\sup X_{-} \notin X$. Consequently, $x \in X_{+}$and by assumption $\inf X_{+}<x$. In particular, there exists some $y \in X_{+}$with $\inf X_{+}<y<x$. Then $y \phi$ is a point in $Y_{+}$satisfying $z<y \phi<\gamma$ for all $z \in Y_{-}$, which contradicts the definition of $\gamma$ as the supremum of $Y_{-}$. We conclude, by symmetry, that neither $\sup Y_{-}$nor $\inf Y_{+}$belong to $Y$. In conclusion, we can now replace $X$ by $Y$ and hence assume that $X$ is the image of the endomorphism $g$.

Our second reduction is to show that we can assume $\alpha=\beta$. Indeed, there is an orderisomorphism $\theta:(\beta, \infty) \rightarrow(\alpha, \infty)$ and we can define a new endomorphism $g^{\prime}$ of $(\mathbb{Q}, \leqslant)$ by

$$
x g^{\prime}= \begin{cases}x g & \text { if } x g \in X_{-} \\ x g \theta & \text { if } x g \in X_{+}\end{cases}
$$

The image of $g^{\prime}$ is order-isomorphic to $X$ and is the disjoint union of $X_{-}$and $X_{+} \theta$. The infimum of $X_{+} \theta$ is also $\alpha$. We may therefore replace $g$ by the endomorphism $g^{\prime}$ and hence assume that $\alpha=\beta$.

In summary, there is an endomorphism $g$ of $(\mathbb{Q}, \leqslant)$ such that the image $\operatorname{im} g=X$ is a disjoint union $X=X_{-} \cup X_{+}$with $X_{-}<X_{+}$and $\sup X_{-}=\inf X_{+}=\alpha \notin X$. Pick any real number $\delta<\alpha$. There is an order-isomorphism $\xi:(-\infty, \alpha) \rightarrow(-\infty, \delta)$ and we extend this to an endomorphism of $(\mathbb{Q}, \leqslant)$ by also defining $x \xi=x$ for all $x \geqslant \alpha$. As $\xi$ is an order-embedding, we conclude that $g \xi \in \operatorname{End}(\mathbb{Q}, \leqslant)$ and $\operatorname{im}(g \xi) \cong X \xi \cong X$. We shall show that $f=g \xi$ is not regular.

Suppose that $h$ is an endomorphism of $(\mathbb{Q}, \leqslant)$ with the property that $f h f=f$. Since $\alpha=\sup X_{-}=\inf X_{+}$, there exist sequences $\left(x_{i}\right)$ and $\left(y_{i}\right)$ in $X_{-}$and $X_{+}$, respectively, 
converging to $\alpha$. As $\xi$ is an order-isomorphism from $(-\infty, \alpha)$ to $(-\infty, \delta)$, we conclude that the sequence $\left(x_{i} \xi\right)$ converges to $\delta$. Pick $q \in \mathbb{Q}$ with $\delta<q<\alpha$. There is a sequence $\left(q_{i}\right)$ in $\mathbb{Q}$ with $q_{i} g=x_{i}$ for each $i$. Now $q_{i} f=x_{i} \xi<\delta<q$, so $x_{i} \xi=q_{i} f=q_{i}$ fhf $\leqslant q h f$ for each $i$. As $\left(x_{i} \xi\right)$ converges to $\delta$, we conclude that $q h g \xi=q h f \geqslant \delta$. From the definition of $\xi$ and the fact that $\alpha \notin \operatorname{im} g=X$, we conclude $q h g>\alpha$.

Similarly, there is a sequence $\left(r_{i}\right)$ in $\mathbb{Q}$ with $r_{i} g=y_{i}$ for each $i$. Now $r_{i} f=y_{i} \xi>\delta>q$, so $y_{i}=y_{i} \xi=r_{i} f=r_{i} f h f \geqslant q h f$ for each $i$. The convergence of $\left(y_{i}\right)$ to $\alpha$, allows us to conclude $q h f \leqslant \alpha$. The definition of $\xi$ forces $q h g<\alpha$.

Comparing the conclusions of the last two paragraphs, we now have a contradiction and hence have established that $f$ is indeed not regular.

Observe that if $X$ is a subset of $\mathbb{Q}$ containing a dense interval, then it satisfies the hypotheses of Theorem 3.10 since we can choose an irrational number $\alpha$ in the corresponding real interval and then partition $X$ into $X_{-}=\{x \in X \mid x<\alpha\}$ and $X_{+}=$ $\{x \in X \mid x>\alpha\}$. On the other hand, if $X \cong(\mathbb{N}, \leqslant)$, then by a similar argument to Proposition 3.8 any endomorphism $f$ with $\operatorname{im} f \cong \mathbb{N}$ is regular.

Corollary 3.11 There are $2^{\aleph_{0}}$ non-regular $\mathscr{D}$-classes in $\operatorname{End}(\mathbb{Q}, \leqslant)$.

Proof: We again make use of the ordered sets $\mathscr{C}_{\mathbf{x}}$ constructed earlier. Let $P$ be the set of enumerations of $\mathbb{Q}$ provided by Proposition 3.5. If $\mathbf{x} \in P$, it is possible to embed a copy of $\mathscr{C}_{\mathbf{x}}$ as a subset $D_{\mathbf{x}}$ of $(2, \infty)$, as $(2, \infty)$ is order-isomorphic to $\mathbb{Q}$. Then take $X_{\mathbf{x}}=$ $(0,1) \cup D_{\mathbf{x}}$. As this set contains an interval, it satisfies the hypotheses of Theorem 3.10 with, for example, $\alpha=\beta=1 / \sqrt{ } 2$ and so there exists a non-regular endomorphism $f_{\mathbf{x}}$ of $(\mathbb{Q}, \leqslant)$ with image isomorphic to $X_{\mathbf{x}}$.

Now if $\mathbf{x}$ and $\mathbf{y}$ are distinct enumerations in $P$, then $X_{\mathbf{x}} \neq X_{\mathbf{y}}$ by Proposition 3.6 combined with the property of $P$. Hence $f_{\mathbf{x}}$ and $f_{\mathbf{y}}$ are not $\mathscr{D}$-related by Lemma 2.1(iii). Thus we do indeed have $2^{\aleph_{0}}$ non-regular $\mathscr{D}$-classes of endomorphisms of $(\mathbb{Q}, \leqslant)$.

\section{Images of idempotent transformations (Theorem B)}

We shall now establish Theorem $\mathrm{B}$, namely that a subset $X$ of $\mathbb{Q}$ arises as the image of an idempotent endomorphism of $(\mathbb{Q}, \leqslant)$ if and only if no maximal interval with the complement of $X$ is closed.

First let $f$ be an idempotent endomorphism of the linearly ordered set of rational numbers $(\mathbb{Q}, \leqslant)$. In order to describe the image of $f$ as a subset of $\mathbb{Q}$ we shall consider the various preimages $x f^{-1}$ of $x \in \mathbb{Q}$. Note that $x f^{-1}$ is empty if $x$ is not in the image of $f$, while $x \in x f^{-1}$ for all $x \in \operatorname{im} f$ because $f$ is idempotent. Define

$$
J=\left\{x \in \operatorname{im} f|| x f^{-1} \mid>1\right\} .
$$

When $f$ is the identity map, $J=\varnothing$ and $\operatorname{im} f=\mathbb{Q}$. For all other idempotent endomorphisms $f, J$ is non-empty and $\operatorname{im} f$ is a proper subset of $\mathbb{Q}$. For the following analysis, we shall assume $f$ is not the identity.

For each $x \in J$, we shall define below two intervals $L_{x}$ and $U_{x}$ in $\mathbb{Q}$. The definition will depend upon the infimum and supremum of the preimage set $x f^{-1}$. If $\inf \left(x f^{-1}\right) \neq-\infty$, then the set $\{q \in \operatorname{im} f \mid q<x\}$ is non-empty. When this set is non-empty and has a maximum member, we shall define

$$
m_{x}=\max \{q \in \operatorname{im} f \mid q<x\} .
$$


Dually, we define

$$
n_{x}=\min \{q \in \operatorname{im} f \mid q>x\}
$$

when this minimum element exists. These two values, when they exist, will contribute to the definition of the intervals $L_{x}$ and $U_{x}$, as follows:

(i) If $x f^{-1}$ is not bounded below, necessarily $x f^{-1}$ contains the interval $(-\infty, x]$. In this case, we define $L_{x}=(-\infty, x)$.

If $x f^{-1}$ is bounded below, then there are three possibilities:

(ii) One possibility is that $\inf \left(x f^{-1}\right)$ is actually an image value of $f$. If so, then $x f^{-1}$ contains the interval $\left(\inf \left(x f^{-1}\right), x\right]$ and necessarily $\inf \left(x f^{-1}\right)$ is the maximum element of $\{q \in \operatorname{im} f \mid q<x\}$; that is, $m_{x}=\inf \left(x f^{-1}\right)$. In this case, we define $L_{x}=\left(m_{x}, x\right)$.

(iii) The next case is that $\inf \left(x f^{-1}\right)$ is not an image value of $f$, but that the element $m_{x}=\max \{q \in \operatorname{im} f \mid q<x\}$ does exist. Necessarily, $m_{x}<\inf \left(x f^{-1}\right)$. In this case, we define $L_{x}=\left(m_{x}, x\right)$. Note that the effect of $f$ on points in $L_{x}$ varies, as follows:

$$
q f= \begin{cases}x & \text { if } \inf \left(x f^{-1}\right)<q<x \\ m_{x} & \text { if } m_{x}<q<\inf \left(x f^{-1}\right)\end{cases}
$$

and, if it is the case that $\inf \left(x f^{-1}\right) \in \mathbb{Q}$, then $\inf \left(x f^{-1}\right) f$ could be either $m_{x}$ or $x$.

(iv) The final case is when $\inf \left(x f^{-1}\right)$ is not an image value of $f$ and there is no maximum element in the set $M=\{q \in \operatorname{im} f \mid q<x\}$. In this case, we define $L_{x}=$ $\left[\inf \left(x f^{-1}\right), x\right)$.

If $\varepsilon>0$, we first observe that there exists some $q \in \operatorname{im} f$ satisfying $\inf \left(x f^{-1}\right)-\varepsilon<$ $q<\inf \left(x f^{-1}\right)$. Indeed, suppose that there were no image value of $f$ belonging to the interval $\left(\inf \left(x f^{-1}\right)-\varepsilon, \inf \left(x f^{-1}\right)\right)$. Choose $r \in \mathbb{Q}$ with $\inf \left(x f^{-1}\right)-\varepsilon<r<\inf \left(x f^{-1}\right)$. Now $r f \neq x$, so $r f \leqslant \inf \left(x f^{-1}\right)$. Since $\inf \left(x f^{-1}\right.$ is not an image value of $f$, our assumption now implies that $r f \leqslant \inf \left(x f^{-1}\right)-\varepsilon<r$. Note that $r f \in M$, so, as the set $M$ has no maximum element, some image value, say $s \in \operatorname{im} f$, satisfies $r f<s<r$. Then $s=s f \leqslant r f$, which is a contradiction. Hence there does indeed exist some $q \in \operatorname{im} f$ satisfying $\inf \left(x f^{-1}\right)-\varepsilon<q<\inf \left(x f^{-1}\right)$. Repeated application of this establishes that in this case there is a monotonic increasing sequence $\left(q_{i}\right)$ of image values of $f$ converging to $\inf \left(x f^{-1}\right)$.

We make a dual set of definitions for $U_{x}$ :

(i) If $x f^{-1}$ is unbounded above, then we set $U_{x}=(x,+\infty)$.

(ii) If $\sup \left(x f^{-1}\right) \in \mathbb{Q}$ is an image value of $f$, then $\sup \left(x f^{-1}\right)=n_{x}$ and we set $U_{x}=$ $\left(x, n_{x}\right)$.

(iii) If $\sup \left(x f^{-1}\right) \in \mathbb{R} \backslash(\operatorname{im} f)$ and $n_{x}=\min \{q \in \operatorname{im} f \mid q>x\}$ exists, then set $U_{x}=$ $\left(x, n_{x}\right)$.

(iv) Otherwise, set $U_{x}=\left(x, \sup \left(x f^{-1}\right)\right]$. In this final case, we can find a sequence of points in $\operatorname{im} f$ converging to $\sup \left(x f^{-1}\right)$.

Lemma 4.1 Let $x \in J$. The intervals $L_{x}$ and $U_{x}$ are either empty or are intervals in $\mathbb{Q}$ that are not closed and are disjoint from the image of $f$. 
Proof: We consider the interval $L_{x}$ in the case when it is non-empty, since the argument for $U_{x}$ is analogous. Since one endpoint is $x \in \mathbb{Q}$ and $x \in \mathbb{Q} \backslash L_{x}$, we see that $L_{x}$ is not closed. We shall now show that it cannot contain a point in the image of $f$.

As $f$ is order-preserving, we know that $q f=x$ for all $q \in\left(\inf \left(x f^{-1}\right), x\right)$. In particular, no point in $\left(\inf \left(x f^{-1}\right), x\right)$ lies in the image of the idempotent map $f$. In particular, this tells us that $L_{x}$ does not meet the image of $f$ in Cases (i), (ii) or (iv) of its definition.

In Case (iii), $m_{x}$ is the maximum element of the image of $f$ satisfying $m_{x}<x$. Consequently, $L_{x}=\left(m_{x}, x\right)$ again does not meet im $f$.

Lemma 4.2 Let $x \in J$. If $L_{x}$ or $U_{x}$ is non-empty, then it is a maximal interval within $\mathbb{Q} \backslash(\operatorname{im} f)$.

Proof: We deal with $L_{x}$ and consider each of the cases (i)-(iv) above in the definition of this interval. The result for $U_{x}$ is established by a dual argument. First note that, by Lemma $4.1, L_{x} \subseteq \mathbb{Q} \backslash(\operatorname{im} f)$. We shall show that $L_{x}$ is a maximal interval in this complement.

In Case (i), $L_{x}=(-\infty, x)$ and the endpoint $x$ belongs to the image of $f$. In Cases (ii) and (iii), $L_{x}=\left(m_{x}, x\right)$ and the endpoints $m_{x}$ and $x$ both belong to im $f$. Hence, in these three cases, $L_{x}$ is a maximal interval within $\mathbb{Q} \backslash(\operatorname{im} f)$.

In Case (iv), $L_{x}=\left[\inf \left(x f^{-1}\right), x\right)$ where $\inf \left(x f^{-1}\right) \notin \operatorname{im} f$. We assume that $\inf \left(x f^{-1}\right)<$ $x$ in order for $L_{x} \neq \varnothing$. The endpoint $x$ belongs to the image of $f$, while there is a sequence $\left(q_{i}\right)$ in $\operatorname{im} f$ converging monotonically to $\inf \left(x f^{-1}\right)$ from below. Hence there cannot exist an interval $I$ contained within $\mathbb{Q} \backslash(\operatorname{im} f)$ that strictly contains $L_{x}$. This completes the proof.

Proof of Theorem B: Let $f$ be any idempotent endomorphism of $(\mathbb{Q}, \leqslant)$, let

$$
J=\left\{x \in \operatorname{im} f|| x f^{-1} \mid>1\right\},
$$

and for each $x \in J$, define the sets $L_{x}$ and $R_{x}$ as described above. Our first step will be to observe that every point in the complement of the image of $f$ belongs to at least one of the sets $L_{x}$ or $R_{x}$ for some $x \in J$.

Let $q \in \mathbb{Q} \backslash(\operatorname{im} f)$ and put $x=q f$. Then $x f^{-1}$ contains $q$, so by definition $x \in J$. Now either $q<x$ or $q>x$. We shall consider the case when $q<x$. The definition tells us that $\inf \left(x f^{-1}\right) \leqslant q$. We now analyse the definition of $L_{x}$ and split into Cases (i)-(iv) as above. In Case (i), $\inf \left(x f^{-1}\right)=-\infty<q$ and so $q \in L_{x}=(-\infty, x)$. In Cases (ii) and (iii), the facts that $f$ is an order-preserving idempotent, $q f=x$ and $m_{x}$ is the maximum image value satisfying $m_{x}<x$ implies that $m_{x}<q$ and so $q \in L_{x}=\left(m_{x}, x\right)$. Finally, in Case (iv), we already know that $\inf \left(x f^{-1}\right) \leqslant q$, so $q \in L_{x}=\left[\inf \left(x f^{-1}\right), x\right)$. Similarly, if $q>x$, then $q \in U_{x}$. In conclusion, every point $q$ not in the image of $f$ lies in either $L_{q f}$ or $U_{q f}$ with $q f \in J$.

We have already observed, in Lemma 4.2 , that the sets $L_{x}$ and $U_{x}$ are maximal intervals in $\mathbb{Q} \backslash(\operatorname{im} f)$ and it now follows, from the previous paragraph, that these sets are all the maximal intervals in $\mathbb{Q} \backslash(\operatorname{im} f)$. We have observed that these sets are not closed in Lemma 4.1. This establishes the necessity part of Theorem B.

Conversely, suppose that $X$ is a subset of $\mathbb{Q}$ and that $\mathbb{Q} \backslash X=\bigcup_{i \in I} T_{i}$, where the sets $T_{i}$, for $i \in I$, are the maximal intervals in $\mathbb{Q} \backslash X$. Assume that the $T_{i}$ are not closed. We define a map $f: \mathbb{Q} \rightarrow \mathbb{Q}$ as follows.

Consider one of the intervals $T_{i}$. Since it cannot be expressed as a closed interval with endpoints $q, r \in \mathbb{R} \cup\{ \pm \infty\}$, it has one of the following forms: 
(i) $T_{i}=[q, r)$ for some $q$ and $r$ with necessarily $r \in \mathbb{Q}$. In this case, define $x f=r$ for all $x \in[q, r)$.

(ii) $T_{i}=(q, r]$ for some $q$ and $r$ with necessarily $q \in \mathbb{Q}$. In this case, define $x f=q$ for all $x \in(q, r]$.

(iii) $T_{i}=(q, r)$ for some $q$ and $r$. Note that at least one of $q$ or $r$ is rational, since otherwise we could write $T_{i}=[q, r]$ contrary to the assumption that $T_{i}$ is not a closed interval. We then define $f$ on this interval depending upon which endpoint is rational:

$$
x f= \begin{cases}q & \text { when } q \in \mathbb{Q} \\ r & \text { when } q \notin \mathbb{Q}\end{cases}
$$

for all $x \in(q, r)$.

Finally define $x f=x$ for all $x \in X$. In this way, we have defined $f$ on the whole set $\mathbb{Q}$. To verify that $f$ is an idempotent endomorphism of $(\mathbb{Q}, \leqslant)$ with image equal to $X$, we now proceed as follows.

First, if $T_{i}=[q, r)$ is a maximal interval in $\mathbb{Q} \backslash X$ with $r \in \mathbb{Q}$, then $r$ cannot belong to another maximal interval $T_{j}$ (as otherwise $T_{i} \cup T_{j}$ would be a larger interval in $\mathbb{Q} \backslash X$ ). Hence $r$ belongs to the set $X$. Similar arguments apply to the other cases in the definition of $f$, so we conclude that $\operatorname{im} f=X$. As a consequence, since $x f=x$ for all $x \in X$, it now follows that $f$ is idempotent.

Finally, we observe that the $f$ is an endomorphism of $(\mathbb{Q}, \leqslant)$. Let $x, y \in \mathbb{Q}$ satisfy $x<y$. When $x, y \in X$, there is nothing to establish since $x f=x$ and $y f=y$. Suppose that $x \in T_{i}$ for some $i$ and that $y \in X$. Let the endpoints of $T_{i}$ be $q$ and $r$ with $q<r$. Then necessarily $q<r \leqslant y$. Our definition for $f$ states that $x f$ equals one of $q$ or $r$. Either way, we know $x f \leqslant r \leqslant y=y f$. A similar argument applies when $x \in X$ and $y \in T_{i}$ for some $i$.

The remaining case is when both $x$ and $y$ lie in one of the maximal intervals $T_{i}$. If they lie in the same maximal interval, then $x f=y f$. If, say, $x \in T_{i}$ and $y \in T_{j}$ with $i \neq j$, let the endpoints of $T_{i}$ and of $T_{j}$ be $q_{1}, r_{1}$ and $q_{2}, r_{2}$, respectively. Then $q_{1}<r_{1} \leqslant q_{2}<r_{2}$. The definition of $f$ tells us $x f \in\left\{q_{1}, r_{1}\right\}$ and $y f \in\left\{q_{2}, r_{2}\right\}$ and $x f \leqslant y f$ follows. Hence $f$ is indeed an idempotent endomorphism of $(\mathbb{Q}, \leqslant)$ with image equal to the set $X$.

This completes the proof of Theorem B.

\section{Countable automorphism groups of countable linearly or- dered structures (Theorem C)}

Let $\Omega=(V, \leqslant)$ where $V$ is a countable set and $\leqslant$ is a linear order on $V$. Throughout this section, we assume that Aut $\Omega$ is a countable group. Our goal in this section is to show that this group is free abelian of finite rank.

Observe that if $X$ is a convex subset of $V$, then every automorphism $\phi$ of $(X, \leqslant)$ can be extended to an automorphism of $\Omega$ by defining

$$
v \hat{\phi}= \begin{cases}v \phi & \text { for } v \in X \\ v & \text { for } v \in V \backslash X .\end{cases}
$$

Thus Aut $X$ embeds as a subgroup of Aut $\Omega$ and so our assumption implies that Aut $X$ is countable for every convex subset $X$ of $V$. We shall use this and similar ideas throughout our argument in this section. 
If $f \in$ Aut $\Omega$, define a relation $\sim$ on $V$ by $x \sim y$ if and only if $x f^{m} \leqslant y \leqslant x f^{n}$ for some $m, n \in \mathbb{Z}$. (Note that $\sim$ depends upon the automorphism $f$, but for simplicity of notation we choose not to write $\sim_{f}$ for this relation.) Then $\sim$ is an equivalence relation on $V$ and we define the orbital $U_{f}(x)$ (following Truss [14]) to be the equivalence class of the point $x$ under the relation $\sim$. Observe that if $x$ is fixed by $f$, then $U_{f}(x)=\{x\}$, while if $x f \neq x$ then the values $x f^{n}$, as $n$ ranges through $\mathbb{Z}$, are distinct and it then follows from the definition that $U_{f}(x)$ is an infinite convex subset of $V$.

The following contains the basic properties of orbitals that we shall need.

Lemma 5.1 Let $f, g \in$ Aut $\Omega$ and $x \in V$.

(i) If $x f>x$, then $U_{f}(x)$ is infinite and $u f>u$ for all $u \in U_{f}(x)$.

(ii) If $x f<x$, then $U_{f}(x)$ is infinite and $u f<u$ for all $u \in U_{f}(x)$.

(iii) $U_{f}(x) g=U_{g^{-1} f g}(x g)$.

(iv) Only finitely many of the orbitals $U_{f}(y)$, as $y$ ranges through $V$, are infinite.

(v) If $f$ and $g$ commute and $U_{f}(x)$ is infinite, then $U_{f}(x) g=U_{f}(x)$.

Proof: (i) We have already observed that if $x f \neq x$, then the orbital $U_{f}(x)$ is infinite. Suppose $x f>x$, then $x f^{n+1}>x f^{n}$ for all $n \in \mathbb{Z}$. So if $u \in U_{f}(x)$, there exist $m, n \in \mathbb{Z}$ such that $x f^{m}<u<x f^{n}$ where necessarily $m<n$. Then $u<x f^{n}<u f^{n-m}$, which can only hold if $u f>u$.

Part (ii) is obtained by a similar argument to (i), while part (iii) is straightforward to establish from the definition.

(iv) Let $\left\{U_{i} \mid i \in I\right\}$ be the set of those orbitals of $f$ that are infinite and suppose that $I$ is infinite. Since the $U_{i}$ are pairwise disjoint and each is a convex subset of $V$, we can define, for each subset $\Sigma$ of $I$, an automorphism $f_{\Sigma}$ of $\Omega$ by

$$
v f_{\Sigma}= \begin{cases}v f & \text { if } v \in U_{i} \text { where } i \in \Sigma \\ v & \text { otherwise. }\end{cases}
$$

Since $f$ induces a non-identity transformation of each $U_{i}$, we conclude that the $f_{\Sigma}$ are distinct. Hence, as $I$ has uncountably many subsets, we obtain a contradiction to the assumption that Aut $\Omega$ is countable. This establishes that only finitely many of the orbitals of $f$ can be infinite.

(v) If $f$ and $g$ commute, then part (iii) of the lemma tells us that the action of $g$ on $V$ induces a permutation on the set of orbitals of $f$. Since only finitely many of these orbitals are infinite and since $g$ preserves the order on $V$, it must be the case that $g$ fixes (setwise) all the orbitals of $f$ that are infinite.

Lemma 5.2 Let $f \in$ Aut $\Omega, x \in V$ and suppose that the orbital $U_{f}(x)$ is infinite. If $a, b \in U_{f}(x)$ with $a<b$, then $\operatorname{Aut}(a, b)=\mathbf{1}$.

Proof: Write $B$ for the interval $(a, b)=\{v \in V \mid a<v<b\}$. Since $a, b \in U_{f}(x)$, there exists some $m \in \mathbb{Z}$ such that $b<a f^{m}$. It follows that the sets $B f^{k m}$, as $k$ ranges over the positive integers, are pairwise disjoint. As $f$ is an automorphism of $\Omega$, each set $B f^{k m}$ is order-isomorphic to $B$. We now have an infinite number of pairwise disjoint convex subsets and so it follows that we can embed the Cartesian product $\prod_{k=0}^{\infty}$ Aut $B f^{k m}$ in Aut $\Omega$ by extending automorphisms defined on each of the sets $B f^{k m}$ to the whole set $V$. In view of the fact that Aut $\Omega$ is countable, we deduce that Aut $B=\mathbf{1}$. 
We are now able to establish one of our main steps along the way to proving Theorem $\mathrm{C}$, namely that the infinite orbitals $U_{f}(x)$, as $f$ ranges over all automorphisms of $\Omega$ and $x$ ranges over $V$, are either disjoint or are equal.

Proposition 5.3 Let $f$ and $g$ be automorphisms of $\Omega, x \in V$ and suppose that both orbitals $U_{f}(x)$ and $U_{g}(x)$ are infinite. Then $U_{f}(x)=U_{g}(x)$.

Proof: Suppose that $U_{f}(x) \neq U_{g}(x)$. If $f$ has more than one infinite orbital, replace $f$ by the map given by

$$
v \tilde{f}= \begin{cases}v f & \text { if } v \in U_{f}(x), \\ v & \text { otherwise. }\end{cases}
$$

Thus, we can assume that $f$ acts as the identity on $V \backslash U_{f}(x)$ and that $U_{f}(v)=\{v\}$ for all $v \in V \backslash U_{f}(x)$. Also, replacing $f$ by $f^{-1}$ if necessary, we can assume that $v f>v$ for all $v \in U_{f}(x)$. Similarly, we can assume that $g$ has only one infinite orbital, namely $U_{g}(x)$, and that $v g>v$ for all $v \in U_{g}(x)$. We deal first with the possibility that one of these infinite orbitals is a subset of the other. Without loss of generality, suppose $U_{f}(x) \subset U_{g}(x)$. We shall consider the possible arrangements of the points in the complement $U_{g}(x) \backslash U_{f}(x)$.

First, if there exist $a, b \in U$ such that $a<U_{f}(x)<b$, then note that $f$ induces a nontrivial automorphism of the interval $(a, b)$. (Indeed, $f$ acts non-trivially on the set $U_{f}(x)$ and fixes all points in $(a, b) \backslash U_{f}(x)$.) We then obtain a contradiction since Lemma 5.2 applied to the orbital $U_{g}(x)$ tells us that $\operatorname{Aut}(a, b)$ is trivial. Hence no such pair $a$ and $b$ exists.

Therefore, if $U_{f}(x) \subset U_{g}(x)$, there exist points in $U_{g}(x)$ greater than those in $U_{f}(x)$ under the order $\leqslant$, or points less than those in $U_{f}(x)$, but not both. The argument for both cases is the same, so we shall assume the existence of some $b \in U_{g}(x)$ with $U_{f}(x)<b$, but that there is no $a \in U_{g}(x)$ with $a<U_{f}(x)$. In this setting, note first that if it were the case that $f$ and $g$ commute, then $U_{f}(x) g=U_{f}(x)$ by Lemma $5.1(\mathrm{v})$, but this contradicts the fact that there exists some $m$ such that $x g^{m}>b$. Hence $f$ and $g$ do not commute.

Now for each $v \in U_{g}(x)$, there is some $n \in \mathbb{Z}$ satisfying $v g^{n}>b$ and so $v g^{n} \notin U_{f}(x)$. Equally, $v g^{m}<x$ for some $m \in \mathbb{Z}$ and so $v g^{m} \in U_{f}(x)$ since $v g^{m}$ cannot satisfy $v g^{m}<$ $U_{f}(x)$. Then $v g^{m}<v g^{n}$, so that $m<n$. It follows that for every $v \in U_{g}(x)$ there is a minimum integer $m(v)$ satisfying $v g^{m(v)} \notin U_{f}(x)$ and this integer has the property that $v g^{n} \in U_{f}(x)$ for all $n<m(v)$ and $v g^{n} \notin U_{f}(x)$ for all $n \geqslant m(v)$.

Now consider the automorphism $\theta_{i}$ defined by $\theta_{i}=g^{i} f g^{-i}$, which by Lemma 5.1(iii) has a single infinite orbital, namely $U_{\theta_{i}}\left(x g^{-i}\right)=U_{f}(x) g^{-i}$, which is some subset of $U_{g}(x)$ (since $U_{f}(x) \subseteq U_{g}(x)$ and $g$ fixes $U_{g}(x)$ setwise). If $v \in U_{g}(x)$, observe $v \in U_{f}(x) g^{-i}$ if and only if $v g^{i} \in U_{f}(x)$; that is, when $i<m(v)$. Consequently, $v \theta_{i}=v$ whenever $i \geqslant m(v)$ and, by Lemma 5.1(i), $v \theta_{i} \neq v$ whenever $i<m(v)$.

Now if $\Sigma=\left\{\sigma_{0}, \sigma_{1}, \sigma_{2}, \ldots\right\}$ is an infinite subset of $\mathbb{N}$ with $\sigma_{i}<\sigma_{i+1}$ for each $i$, we can define another automorphism of $\Omega$ by

$$
h_{\Sigma}=\lim _{n \rightarrow \infty} \theta_{\sigma_{n}} \ldots \theta_{\sigma_{1}} \theta_{\sigma_{0}} .
$$

(In order to make sense of this definition, recall our convention is to write maps on the right.) If $v \in V \backslash U_{g}(x)$, then $v \theta_{i}=v$ for all $i$, so we observe $v \theta_{\sigma_{n}} \ldots \theta_{\sigma_{0}}=v$ and hence $v h_{\Sigma}$ is defined and indeed equals $v$ for such $v$. On the other hand, if $v \in U_{g}(x)$, then there exists some $N$ such that $\sigma_{m} \geqslant m(v)$ for all $m>N$. Thus $v \theta_{\sigma_{m}}=v$ for all such $m$ and we conclude that

$$
v \theta_{\sigma_{n}} \ldots \theta_{\sigma_{1}} \theta_{\sigma_{0}}=v \theta_{\sigma_{N}} \ldots \theta_{\sigma_{1}} \theta_{\sigma_{0}}
$$


for all $n>N$. Hence $v h_{\Sigma}$ is defined for all $v \in U_{g}(x)$ since $v \theta_{\sigma_{n}} \ldots \theta_{\sigma_{0}}$ takes the same value independent of $n$ provided this $n$ is large enough. In addition to having observed that $h_{\Sigma}$ is well-defined, such calculations similarly show that $h_{\Sigma} \in$ Aut $\Omega$.

Having verified that $h_{\Sigma}$ is defined for any (infinite) $\Sigma \subseteq \mathbb{N}$, we now observe that $h_{\Sigma} \neq h_{T}$ for distinct $\Sigma, T \subseteq \mathbb{N}$. Indeed, suppose $\Sigma=\left\{\sigma_{0}, \sigma_{1}, \ldots, \sigma_{r-1}, \sigma_{r}, \ldots\right\}$ and $T=\left\{\sigma_{0}, \sigma_{1}, \ldots, \sigma_{r-1}, \tau_{r}, \ldots\right\}$ where, without loss of generality, $\sigma_{r}<\tau_{r}$. Take $u=x g^{k}$ where $k=m(x)-\sigma_{r}-1$. Observe $u g^{\sigma_{r}}=x^{m(x)-1} \in U_{f}(x)$ and $u g^{i} \notin U_{f}(x)$ for all $i>\sigma_{r}$. Thus $u \notin U_{f}(x) g^{-i}$ for all $i>\sigma_{r}$, so that $u \theta_{i}=u$ for all such $i$. Hence, for $n \geqslant r$,

$$
u \theta_{\sigma_{n}} \ldots \theta_{\sigma_{0}}=u \theta_{\sigma_{r}} \ldots \theta_{\sigma_{0}} \quad \text { and } \quad u \theta_{\tau_{n}} \ldots \theta_{\tau_{r}} \theta_{\sigma_{r-1}} \ldots \theta_{\sigma_{0}}=u \theta_{\sigma_{r-1}} \ldots \theta_{\sigma_{0}} .
$$

As $u \in U_{f}(x) g^{-\sigma_{r}}=U_{\theta_{\sigma_{r}}}(x)$, we know $u \theta_{\sigma_{r}} \neq u$ and so we conclude $u h_{\Sigma} \neq u h_{T}$, which establishes our claim that the $h_{\Sigma}$ are distinct. Since Aut $\Omega$ is countable, it cannot contain these uncountably many automorphisms $h_{\Sigma}$ and we have another contradiction. The other remaining case when $U_{f}(x) \subset U_{g}(x)$ is similar, which now establishes that $U_{f}(x)$ is not a subset of $U_{g}(x)$ nor vice versa.

Thus there exists some $a \in U_{f}(x)$ and $b \in U_{g}(x)$ such that $a \notin U_{g}(x)$ and $b \notin U_{f}(x)$. We may assume, without loss of generality that $a<U_{g}(x)$. Then, since $U_{f}(x)$ and $U_{g}(x)$ are convex, we observe $U_{f}(x)<b$. Moreover we also note that the sets $U_{f}(x) \backslash U_{g}(x)$, $U_{f}(x) \cap U_{g}(x)$ and $U_{g}(x) \backslash U_{f}(x)$ are all convex and satisfy $U_{f}(x) \backslash U_{g}(x)<U_{f}(x) \cap U_{g}(x)<$ $U_{g}(x) \backslash U_{f}(x)$. Suppose first that $f$ and $g$ commute. Then as $b, x \in U_{g}(x)$, there exists some $n \in \mathbb{Z}$ such that $b<x g^{n}$. However, this is impossible as $x g^{n} \in U_{f}(x)$ by use of Lemma $5.1(\mathrm{v})$. Hence we it must be the case that $f$ and $g$ do not commute. Put $h=f^{-1} g^{-1} f g$, which is some non-identity element of Aut $\Omega$. If $v \in U_{f}(x) \backslash U_{g}(x)$, then $v f^{-1}<v<U_{g}(x)$ and so $v f^{-1} \notin U_{g}(x)$ and hence $v f^{-1} g^{-1} f g=v f^{-1} f g=v g=v$. Similarly, if $v h=v$ for $v \in U_{g}(x) \backslash U_{f}(x)$. It follows that any infinite $U_{h}(y)$ is a subset of $U_{f}(x) \cap U_{g}(x)$. However, we have already established that this is impossible, since a pair of non-identity automorphisms $f$ and $h$ cannot have infinite orbitals satisfying $U_{h}(x) \subset U_{f}(x)$. This final contradiction completes the proof of the claim: $U_{f}(x)=U_{g}(x)$.

Recall that a linearly order group is a group $G$ together with a linear order $\leqslant$ upon it such that if $g, h, k \in G$ with $h \leqslant k$, then $g h \leqslant g k$ and $h g \leqslant k g$. An Archimedean group is a linearly ordered group $G$ with the property if $g, h \in G$ satisfy $1<g<h$, there exists $n \in \mathbb{N}$ such that $h<g^{n}$.

Let $f \in$ Aut $\Omega$ and fix $x \in V$ such that the orbital $U=U_{f}(x)$ is infinite. For $\phi, \psi \in$ Aut $U$, define $\phi \leqslant \psi$ whenever $x \phi \leqslant x \psi$. We shall observe that this is a welldefined linear order with respect to which Aut $U$ is an Archimedean group.

Lemma 5.4 (i) The map $\xi$ : Aut $U \rightarrow U$ given by $\phi \mapsto x \phi$ for each $\phi \in$ Aut $U$ is an injective map.

(ii) The order $\leqslant$ is a well-defined linear order on $\operatorname{Aut} U$ with respect to which $\operatorname{Aut} U$ is an Archimedean group.

Proof: (i) Suppose $\phi$ and $\psi$ are distinct automorphisms of $U$. Then $g=\phi \psi^{-1}$ can be extended to a non-identity automorphism of $\Omega$ by defining $v g=v$ for all $v \in V \backslash U$. By assumption some $u \in U$ is moved by $g$ and then $U_{g}(u)=U$ by Proposition 5.3. In particular, $x \in U_{g}(u)$ and hence $x g \neq x$ by Lemma 5.1(i)-(ii). This shows that $x \phi \neq x \psi$, as is required to establish that $\xi$ is injective. 
(ii) Part (i) of the lemma shows that the set of automorphisms of $U$ is in one-one correspondence with the subset $\{x \phi \mid \phi \in \operatorname{Aut} U\}$ of $U$ and hence the order on $U$ induces an order on Aut $U$; that is, the order $\leqslant$ defined by $\phi \leqslant \psi$ if and only if $x \phi \leqslant x \psi$. It is straightforward to verify that Aut $U$ is a linearly ordered group with respect to $\leqslant$. (One makes use of Lemma 5.1 in this verification. For example, if $\phi, \psi, \theta \in$ Aut $U$ with $\phi \leqslant \psi$, then $x \psi \phi^{-1} \geqslant x$ and use of Lemma 5.1(ii) shows that $x \theta \psi \phi^{-1} \geqslant x \theta$. It then follows $\theta \phi \leqslant \theta \psi$, which is one of the facts that needs to be established.)

Finally, if $1<\phi<\psi$, we extend $\phi$ to an isomorphism of $\Omega$ and observe $U=U_{\phi}(x)$ by Proposition 5.3. The definition of $U_{\phi}(x)$ then provides $n \in \mathbb{N}$ such that $x \psi<x \phi^{n}$, so $\psi<\phi^{n}$. This establishes that Aut $U$ is an Archimedean group with respect to the order $\leqslant$.

We can now make use of the result, originally due to Hölder [9], that an Archimedean group is isomorphic to an additive subgroup of the set $\mathbb{R}$ of real numbers (see, for example, [6, Theorem 4.A]). In [7, Lemma 4.21] it is noted that such a subgroup is either cyclic or is a dense subset of $\mathbb{R}$. Our current goal is to establish Proposition 5.9 below, namely that Aut $U$ is an infinite cyclic group, so let us assume, seeking a contradiction, that $($ Aut $U, \leqslant)$ is a dense linearly ordered set.

In Lemma 5.4 we have observed that the map $\xi$ is an order-isomorphism from $\operatorname{Aut} U$ to the orbit of $x$ under the action of Aut $U$ (with the order on this orbit being that induced from the ordered set $\Omega$ ). Thus $\{x \phi \mid \phi \in$ Aut $U\}$ is a dense linearly ordered set with no maximum or minimum element and is therefore order-isomorphic to $(\mathbb{Q}, \leqslant)$. This observation is independent of the choice of representative $x$ in $U$ and hence every orbit in $U$ under the action of Aut $U$ is order-isomorphic to $(\mathbb{Q}, \leqslant)$.

Lemma 5.5 If $u, v \in U=U_{f}(x)$ and $\phi, \psi \in$ Aut $U$ with $\phi<\psi$, then there exists $\theta \in$ Aut $U$ with $u \phi<v \theta<u \psi$.

Proof: By use of Lemma 5.1(ii), we observe that the hypothesis $\phi<\psi$ ensures that $u \phi<u \psi$. When $u$ and $v$ belong to the same orbit of Aut $U$ on $U$ the claim is now immediate since that orbit is order-isomorphic to $(\mathbb{Q}, \leqslant)$. Suppose that $v$ is not in the orbit of $u$ under the action of Aut $U$ and, by applying $\phi^{-1}$ if necessary, assume that $\phi$ is the identity automorphism. Thus $\psi$ is a non-identity automorphism of $U$ satisfying $u<u \psi$ and we must find $\theta \in \operatorname{Aut} U$ with $u<v \theta<u \psi$.

Suppose first that $v<u$. We extend $\psi$ to an automorphism of $\Omega$ by defining it to fix all points outside the orbital $U$. Then Proposition 5.3 tells us that $U_{\psi}(u)=U_{f}(u)=U$. In particular, there exists some $n \in \mathbb{N}$ such that $v \psi^{n}>u$. Take $n$ to be the minimum positive integer satisfying $v \psi^{n}>u$. Then $v \psi^{n-1}<u$, so $u<v \psi^{n}<u \psi$ and so, in this case, $\theta=\psi^{n}$ is our required automorphism.

If $u<v$, then since $U_{\psi}(u)=U$ we can find some power of $\psi$ such that $v \psi^{m}<u$. Applying the previous paragraph to $v \psi^{m}$ finds $n \in \mathbb{N}$ such that $u<v \psi^{m+n}<u \psi$ and then $\theta=\psi^{m+n}$ is the automorphism we seek.

Now enumerate the points in $U$ as the sequence $\left(x_{n}\right)$. First consider the set $\mathcal{T}_{0}$ of convex subsets $S$ of $U$ such that (i) $S$ contains $x_{0}$ and (ii) $S \phi$ is disjoint from $S$ for every non-identity automorphism $\phi$ of $U$. As only the identity automorphism fixes $x_{0}$ (see Lemma 5.1(i)-(ii)) we conclude $\left\{x_{0}\right\}$ is a set in $\mathcal{T}_{0}$ (so $\mathcal{T}_{0}$ is non-empty) and it is straightforward to verify that the union of any chain of subsets of $\mathcal{T}_{0}$ is again a member of $\mathcal{T}_{0}$. Hence, by Zorn's Lemma, there is some maximal member $M_{0}$ of $\mathcal{T}_{0}$.

Suppose then that, for some $n$, we have found subsets $M_{0}, M_{1}, \ldots, M_{k}$ of $U$ such that 
- $x_{0}, x_{1}, \ldots, x_{n-1} \in\left\{u \phi \mid u \in M_{0} \cup \cdots \cup M_{k}, \phi \in \operatorname{Aut} U\right\}$, and

- $M_{i}$ is a maximal convex subset of $U$ subject to

$$
\begin{aligned}
& M_{i} \subseteq U \backslash\left\{u \phi \mid u \in M_{0} \cup \cdots \cup M_{i-1}, \phi \in \text { Aut } U\right\} \\
& M_{i} \phi \cap M_{i}=\varnothing \text { for every non-identity automorphism } \phi \text { of } U .
\end{aligned}
$$

(Note that Condition (1) ensures that $M_{i}$ is disjoint from every translate $M_{j} \phi$ of a previously defined subset, with $1 \leqslant j<i$, under some automorphism of $U$.)

If $x_{n}$ is already the image of some point in $M_{0} \cup \cdots \cup M_{k}$ under some automorphism of $U$, then we need create no new subset $M_{i}$ at this stage. Otherwise, consider the set $\mathcal{T}_{k+1}$ of subsets $S$ of $U \backslash\left\{u \phi \mid u \in M_{0} \cup \cdots \cup M_{k}, \phi \in \operatorname{Aut} U\right\}$ such that (i) $S$ is a convex subset of $U$, (ii) $x_{n} \in S$, and (iii) $S \phi$ is disjoint from $S$ for every non-identity automorphism $\phi$ of $U$. Again, an application of Zorn's Lemma provides the existence of a maximal member $M_{k}$ in $\mathcal{T}_{k+1}$.

In this way, we find a family $\left(M_{i}\right)$ of convex subsets of $U$, indexed by some set $I$ (where either $I=\mathbb{N}$ or $I=\{0,1, \ldots, k\}$ for some $k$ ), such that $U$ is the disjoint union of the sets $M_{i} \phi$, for $i \in I$ and $\phi \in \operatorname{Aut} U$, and $M_{i}$ is maximal among convex subsets of $U$ satisfying (1) and (2) above. As convex subsets of the linearly ordered set $U$, there is an induced order on the sets $\left\{M_{i} \phi \mid i \in I, \phi \in \operatorname{Aut} U\right\}$.

Lemma 5.6 Suppose that $M_{i_{1}} \phi_{1}<M_{i_{2}} \phi_{2}$ for some $i_{1}, i_{2} \in I$ and some $\phi_{1}, \phi_{2} \in$ Aut $U$. Then for each $j \in I$, there exists some $\psi \in \operatorname{Aut} U$ with

$$
M_{i_{1}} \phi_{1}<M_{j} \psi<M_{i_{2}} \phi_{2} .
$$

Proof: Suppose first that $i_{1}=i_{2}$. Pick $u \in M_{i_{1}}$ and $v \in M_{j}$. By Lemma 5.5, there exists $\psi \in$ Aut $U$ such that $u \phi_{1}<v \psi<u \phi_{2}$. Hence, as the sets concerned are convex, $M_{i_{1}} \phi_{1}<M_{j} \psi<M_{i_{1}} \phi_{2}=M_{i_{2}} \phi_{2}$, as required.

It remains to deal with the case when $i_{1} \neq i_{2}$. If there exists some $k \in I$ and automorphisms $\theta_{1}, \theta_{2} \in$ Aut $U$ with $M_{i_{1}} \phi_{1} \leqslant M_{k} \theta_{1}<M_{k} \theta_{2} \leqslant M_{i_{2}} \phi_{2}$, then the previous paragraph can be applied to $M_{k} \theta_{1}<M_{k} \theta_{2}$ and we would have established the required result. Seeking a contradiction, let us assume that no such $k, \theta_{1}$ and $\theta_{2}$ exist. As a consequence, we conclude that there is no $\theta \in$ Aut $U$ with $M_{i_{1}} \phi_{1}<M_{i_{1}} \theta<M_{i_{2}} \phi_{2}$ or with $M_{i_{1}} \phi_{1}<M_{i_{2}} \theta<M_{i_{2}} \phi_{2}$ and that, for each $k \in I$, there is at most one $\theta \in \operatorname{Aut} U$ with $M_{i_{1}} \phi_{1}<M_{k} \theta<M_{i_{2}} \phi_{2}$.

Write $K$ for the set of those $k \in I$ for which there exists $\theta_{k} \in$ Aut $U$ with $M_{i_{1}} \phi_{1} \leqslant$ $M_{k} \theta_{k} \leqslant M_{i_{2}} \phi_{2}$. (So, in particular, $i_{1}, i_{2} \in K$ and that $\theta_{i_{m}}=\phi_{m}$ for $m=1,2$.) Let $m$ be the smallest integer in $K$. By applying the inverse of $\theta_{m}$ if necessary, there is no loss of generality in assuming that $\theta_{m}$ is the identity map. Put $S=\bigcup_{k \in K} M_{k} \theta_{k}$, so that $M_{m}$ is a proper subset of $S$ by our assumption on $\theta_{m}$. Since $V$ is the union of all translates $M_{j} \theta$, it follows that every point between $M_{i_{1}} \phi_{1}$ and $M_{i_{2}} \phi_{2}$ lies in some $M_{k} \theta_{k}$ with $k \in K$ and we deduce that the set $S$ is convex. The set $S$ is also disjoint from all translates of $M_{j}$ for $j<m$, since each set $M_{k}$ for $k \in K$ satisfies (1) above, while $S \psi \cap S=\varnothing$ for every nonidentity $\psi \in \operatorname{Aut} U$ since each set $M_{k}$ satisfies (2). We now have a contradiction to $M_{m}$ being a maximal convex subset satisfying (1) and (2). This contradiction completes the proof of the lemma.

The property given in Lemma 5.6 will essentially characterise the structure of the ordered set $(U, \leqslant)$. To describe this fully, we first introduce a new relational structure. 
Let $I$ be a countable set. We define an $I$-coloured linearly ordered set to be a relational structure $\Omega=\left(V, \leqslant,\left(R_{i}\right)_{i \in I}\right)$ where $\leqslant$ is a linear order on the set $V$ and where each $R_{i}$ is a binary relation on $V$ of the form $R_{i}=V_{i} \times V_{i}$ such that $V$ is the disjoint union of the sets $V_{i}$. Thus the sequence $\left(R_{i}\right)_{i \in I}$ encodes an equivalence relation on $V$ with equivalence classes $V_{i}$, for $i \in I$, in such a way that any automorphism of $\Omega$ fixes each of the equivalence classes setwise.

The class of finite $I$-coloured linearly ordered sets satisfies the hereditary property, the joint embedding property and the amalgamation property and therefore this class possesses a unique Fraïssé limit $\mathbb{Q}_{I}=\left(W, \leqslant,\left(R_{i}\right)_{i \in I}\right)$. Write $W_{i}$ for the equivalence class determined by the relation $R_{i}$. This structure is characterised by the following property: $(W, \leqslant)$ is a countable linearly ordered set without maximum or minimum elements such that for every pair $u, v \in W$ with $u<v$ and every $i \in I$ there exists $w \in W_{i}$ with $u<w<v$. Indeed, it can be shown by a back-and-forth argument that any two countable structures satisfying this condition are isomorphic as $I$-coloured linearly ordered sets (and again such an isomorphism takes the equivalence class in the first structure indexed by $i \in I$ to that in the second indexed by $i$ ). We shall call this Fraïssé limit the $I$-coloured ordered set of rational numbers in view of the fact that $(W, \leqslant)$ is order-isomorphic to $(\mathbb{Q}, \leqslant)$. In view of this order-isomorphism, we shall rename the set $W$ as $\mathbb{Q}$, so that the $I$-coloured linearly ordered set is denoted $\mathbb{Q}_{I}=\left(\mathbb{Q}, \leqslant,\left(R_{i}\right)_{i \in I}\right)$.

Proposition 5.7 The automorphism group of the I-coloured ordered set $\mathbb{Q}_{I}$ of rational numbers is uncountable.

Proof: Note that Aut $\mathbb{Q}_{I}$ is non-trivial since given any $i \in I$ and two points $x, y \in W_{i}$, a back-and-forth argument establishes the existence of an order-isomorphism that preserves the equivalence classes $W_{i}$ and maps $x$ to $y$. The following argument extends this to show in fact there are uncountably many automorphisms of $\mathbb{Q}_{I}$.

We shall write $\mathbb{Z} \times \mathbb{Q}_{I}$ for the $I$-coloured linearly ordered set defined as follows: as an ordered set it is the set $\mathbb{Z} \times \mathbb{Q}$ equipped with the lexicographic order; that is, $(m, x) \leqslant(n, y)$ if and only if $m<n$, or $m=n$ and $x \leqslant y$. To colour $\mathbb{Z} \times \mathbb{Q}_{I}$, for each $i \in I$, the $i$ th equivalence class is $\mathbb{Z} \times W_{i}$ where $W_{i}$ is the $i$ th equivalence class in $\mathbb{Q}_{I}$. In effect, with $\mathbb{Z} \times \mathbb{Q}_{I}$, we are taking countably many copies of $\mathbb{Q}_{I}$, placing them in sequence in terms of the order, and then taking the $i$ th equivalence classes in each copy of $\mathbb{Q}_{I}$ together to form a single equivalence class in $\mathbb{Z} \times \mathbb{Q}_{I}$.

One observes that $\mathbb{Z} \times \mathbb{Q}_{I}$ is a countable linearly ordered set with no maximum or minimum element and that it has the property that for each $u, v \in \mathbb{Z}$ with $u<v$ and all $i \in I$, there exists $w \in \mathbb{Z} \times W_{i}$ with $u<w<v$. Thus, $\mathbb{Z} \times \mathbb{Q}_{I}$ satisfies the defining property of $\mathbb{Q}_{I}$ so that $\mathbb{Z} \times \mathbb{Q}_{I} \cong \mathbb{Q}_{I}$ as $I$-coloured linearly ordered sets.

If $\mathbf{f}=\left(f_{n}\right)$ is a sequence of automorphisms of the structure $\mathbb{Q}_{I}$, we can define $\hat{\mathbf{f}} \in$ $\operatorname{Aut}\left(\mathbb{Z} \times \mathbb{Q}_{I}\right)$ by $(n, x) \hat{\mathbf{f}}=\left(n, x f_{n}\right)$ for each $n \in \mathbb{Z}$ and $x \in \mathbb{Q}$. This defines an injective $\operatorname{map} \mathbf{f} \mapsto \hat{\mathbf{f}}$ from the Cartesian product $\prod_{n=1}^{\infty} \operatorname{Aut} \mathbb{Q}_{I}$ to $\operatorname{Aut}\left(\mathbb{Z} \times \mathbb{Q}_{I}\right)$. It now follows that Aut $\mathbb{Q}_{I} \cong \operatorname{Aut}\left(\mathbb{Z} \times \mathbb{Q}_{I}\right)$ is indeed uncountable.

We now return to the automorphism group of the orbital $U=U_{f}(x)$ under our current assumption that Aut $U$ is order-isomorphic to some dense linearly ordered set. Recall that we have defined a sequence $\mathbf{M}=\left(M_{i}\right)_{i \in I}$ of convex subsets of $U$ indexed by $I$. We shall use the $I$-coloured ordered set $\mathbb{Q}_{I}$ of rational numbers, where $I$ is the set indexing our convex subsets. Recall that the equivalence classes on $\mathbb{Q}$ associated to this relational structure are denoted $\left(W_{i}\right)_{i \in I}$. Now write $\mathbb{Q}_{I}(\mathbf{M})$ for the ordered set $(S, \leqslant)$ where $S=\bigcup_{i \in I}\left(W_{i} \times M_{i}\right)$ and the order $\leqslant$ is the lexicographic order (that is, $(x, m) \leqslant(y, n)$ if and only if $x<y$, 
or $x=y$ and $m \leqslant n$ ). Now if $\phi \in \operatorname{Aut} \mathbb{Q}_{I}$ (a colour- and order-preserving bijection of this structure), we can define an automorphism $\tilde{\phi}$ of $\mathbb{Q}_{I}(\mathbf{M})$ by $(x, m) \tilde{\phi}=(x \phi, m)$ for $(x, m) \in S$. Note that we rely upon the fact that $\phi$ preserves the $i$ th equivalence class $W_{i}$ when observing that $\tilde{\phi}$ is indeed a well-defined map. The following now follows from the fact that Aut $\mathbb{Q}_{I}$ is uncountable.

Corollary 5.8 The automorphism group of the ordered set $\mathbb{Q}_{I}(\mathbf{M})=(S, \leqslant)$ is uncountable.

Let us now consider the set $\mathcal{M}=\left\{M_{i} \phi \mid i \in I, \phi \in\right.$ Aut $\left.U\right\}$ of all translates of the sets $M_{i}$ under the action of the automorphism group of $U$ and view this as an ordered set using the order induced on these convex subsets from the order on $U$. We shall also define a relation $R_{i}$ to be that relating the points $M_{i} \phi$ for $\phi \in \operatorname{Aut} U$, so that $W_{i}=$ $\left\{M_{i} \phi \mid \phi \in\right.$ Aut $\left.U\right\}$ is the corresponding subset of $\mathcal{M}$ indexed by $i$. Since Aut $U$ has no maximum or minimum element, the same is true of $\mathcal{M}$ and now Lemma 5.6 tells us that $\left(\mathcal{M}, \leqslant,\left(R_{i}\right)_{i \in I}\right)$ satisfies the defining property of the $I$-coloured ordered set $\mathbb{Q}_{I}$ of rational numbers. Thus these structures are isomorphic as $I$-coloured ordered sets. Returning to our set $U$, we now observe that we can reconstruct this set from $\mathcal{M}$ by replacing each point $M_{i} \phi$ by a copy of the ordered set $M_{i}$. This tells us that $(U, \leqslant)$ is order-isomorphic to $\mathbb{Q}_{I}(\mathbf{M})$. This now gives us the contradiction that we seek: Corollary 5.8 tells us that Aut $U$ is uncountable, which is contrary to our running assumption.

In conclusion, we have now established our final step towards the theorem.

Proposition 5.9 Let $f \in$ Aut $\Omega, \quad x \in V$ and suppose $U=U_{f}(x)$ is infinite. Then Aut $U$ is an infinite cyclic group.

Putting all our work together, we can now establish Theorem C.

Proof of Theorem C: Consider the set $\left\{U_{i} \mid i \in I\right\}$ of all subsets of $V$ that arise as an infinite orbital of some automorphism of $\Omega$. Proposition 5.3 tells us that these sets $U_{i}$ are pairwise disjoint. Moreover, if $f_{i} \in$ Aut $U_{i}$ for each $i \in I$, then there is an extension $f$ to an automorphism of $\Omega$ by

$$
v f= \begin{cases}v f_{i} & \text { if } v \in U_{i} \text { for some } i \in I, \\ v & \text { otherwise }\end{cases}
$$

Since any automorphism of $\Omega$ must fix all points in $V \backslash\left(\bigcup_{i \in I} U_{i}\right)$, we conclude that Aut $\Omega$ is isomorphic to the Cartesian product of the automorphism groups of the $U_{i}$. The countability of Aut $\Omega$ combined with Proposition 5.9 tells us that $I$ is finite and that Aut $\Omega \cong \mathbb{Z}^{|I|}$. This completes the proof of our theorem.

Acknowledgements: Jillian McPhee was funded by an EPSRC Doctoral Training Grant. Martyn Quick acknowledges support by EPSRC grant EP/H011978/1. The authors thank Igor Dolinka for drawing their attention to the results of Kubis in [11].

\section{References}

[1] Meenaxi Bhattacharjee, Dugald Macpherson, Rögnvaldur Möller \& Peter M. Neumann, Notes on Infinite Permutation Groups, Texts and Readings in Math. 12, Lecture Notes in Math. 1698, Hindustan Book Agency, New Delhi; co-published by Springer-Verlag, Berlin, 1997. 
[2] Johannes de Groot, "Groups represented by homeomorphism groups," Math. Annalen 138 (1959), 80-120.

[3] Igor Dolinka, Robert D. Gray, Jillian D. McPhee, James D. Mitchell \& Martyn Quick, "Automorphism groups of countable algebraically closed graphs and endomorphisms of the random graph," Math. Proc. Cambridge Philos. Soc. 160 (2016), 437-462.

[4] R. Fraïssé, "Sur certains relations qui généralisent l'order des nombres rationnels," C. R. Acad. Sci. Paris 237 (1953), 540-542.

[5] Robert Frucht, "Herstellung von Graphen mit vorgegebener abstrakter Gruppe," Compositio Math. 6 (1939), 239-250.

[6] A. M. W. Glass, Partially Ordered Groups, Series in Algebra 7, World Scientific Publishing Co. Pte. Ltd., Singapore, 1999.

[7] K. R. Goodearl, Partially ordered abelian groups with interpolation, Mathematical Surveys and Monographs 20, Amer. Math. Soc., Providence RI, 1986.

[8] W. Hodges, A Shorter Model Theory, Cambridge University Press, Cambridge, 1997.

[9] O. Hölder, "Die Axiome der Quantität und die Lehre von Mass," Ber. Verh. Sächs. Ges. Wiss. Leipzig Math.-Phys. Cl. 53 (1901), 1-64.

[10] John M. Howie, Fundamentals of Semigroup Theory, London Math. Soc. Monographs New Ser. 12, Oxford University Press, Oxford, 1995.

[11] Wiesław Kubiś, "Injective objects and retracts of Fraïssé limits," Forum Math. 27 (2015), 807-842.

[12] Jillian D. McPhee, Endomorphisms of Fraïssé Limits and Automorphism Groups of Algebraically Closed Relational Structures, PhD thesis, University of St Andrews, 2012 .

[13] Gert Sabidussi, "Graphs with given infinite group," Monat. Math. 64 (1960), 64-67.

[14] J. K. Truss, "Infinite permutation groups. I. Products of conjugacy classes," J. Algebra 120 (1989), 454-493. 